\title{
The solvability cardinal of the class of polynomials
}

\author{
MiKLÓs LACZKOVICH
}

Dedicated to Professor Ludwig Reich on the occasion of his 80th birthday.

\begin{abstract}
Let $G$ be an Abelian group, and let $\mathbb{C}^{G}$ denote the set of complex valued functions defined on $G$. A map $D: \mathbb{C}^{G} \rightarrow \mathbb{C}^{G}$ is a difference operator, if there are complex numbers $a_{i}$ and elements $b_{i} \in G(i=1, \ldots, n)$ such that $(D f)(x)=\sum_{i=1}^{n} a_{i} f\left(x+b_{i}\right)$ for every $f \in \mathbb{C}^{G}$ and $x \in G$. By a system of difference equations we mean a set of equations $\left\{D_{i} f=g_{i}: i \in I\right\}$, where $I$ is an arbitrary set of indices, $D_{i}$ is a difference operator and $g_{i} \in \mathbb{C}^{G}$ is a given function for every $i \in I$, and $f$ is the unknown function. The solvability cardinal sc $(\mathcal{F})$ of a class of functions $\mathcal{F} \subset \mathbb{C}^{G}$ is the smallest cardinal number $\kappa$ with the following property: whenever $S$ is a system of difference equations on $G$ such that each subsystem of $S$ of cardinality $<\kappa$ has a solution in $\mathcal{F}$, then $S$ itself has a solution in $\mathcal{F}$. The behaviour of $\operatorname{sc}(\mathcal{F})$ is rather erratic, even for classes of functions defined on $\mathbb{R}$. For example, sc $(\mathbb{C}[x])=3$, but sc $(\mathcal{T} \mathcal{P})=\omega_{1}$, where $\mathcal{T} \mathcal{P}$ is the set of trigonometric polynomials; sc $\left(\mathbb{C}^{\mathbb{R}}\right)=\omega$, but sc $(\mathcal{D} \mathcal{F})=\left(2^{\omega}\right)^{+}$, where $\mathcal{D} \mathcal{F}$ is the set of functions having the Darboux property. Our aim is to determine or to estimate the solvability cardinal of the class of polynomials defined on $\mathbb{R}^{n}$, on normed linear spaces and, in general, on topological Abelian groups. Let $\mathcal{P}_{G}$ denote the class of polynomials defined on the group $G$. After presenting some general estimates we prove that $\mathrm{sc}\left(\mathbb{C}\left[x_{1}, \ldots, x_{n}\right]\right)=\omega$ if $2 \leq n<\infty$, and $\operatorname{sc}\left(\mathcal{P}_{X}\right)=\omega_{1}$ if $X$ is a normed linear space of infinite dimension. For discrete Abelian groups we show that $\operatorname{sc}\left(\mathcal{P}_{G}\right)=3$ if $r_{0}(G) \leq 1, \operatorname{sc}\left(\mathcal{P}_{G}\right)=\omega$ if $2 \leq r_{0}(G)<\infty$, and $\operatorname{sc}\left(\mathcal{P}_{G}\right) \geq \omega_{1}$ if $r_{0}(G)$ is infinite, where $r_{0}(G)$ denotes the torsion free rank of $G$. The solvability of systems of difference equations is closely connected to the existence of projections of function classes commuting with translations (see Theorem 7.1). As an application we construct a projection from $\mathbb{C}^{\mathbb{R}^{n}}$ onto $\mathbb{C}\left[x_{1}, \ldots, x_{n}\right]$ commuting with translations by vectors having rational coordinates (Theorem 7.4).
\end{abstract}

Mathematics Subject Classification. Primary 39A06, 39A70, 47B39; Secondary 22A10, 13 B25.

Keywords. Polynomials, Systems of difference equations, Topological Abelian groups.

M. Laczkovich: Partially supported by Hungarian Scientific Foundation Grant No. K124749. 


\section{Introduction}

Let $G$ be an Abelian group, and let $\mathbb{C}^{G}$ denote the set of complex valued functions defined on $G$. A map $D: \mathbb{C}^{G} \rightarrow \mathbb{C}^{G}$ is said to be a difference operator, if there are complex numbers $a_{i}$ and elements $b_{i} \in G(i=1, \ldots, n)$ such that $(D f)(x)=\sum_{i=1}^{n} a_{i} f\left(x+b_{i}\right)$ for every $f \in \mathbb{C}^{G}$ and $x \in G$. The set of difference operators on $G$ is denoted by $\mathcal{D}_{G}$.

By a system of difference equations we mean a set of equations $S=\left\{D_{i} f=\right.$ $\left.g_{i}: i \in I\right\}$, where $I$ is an arbitrary set of indices, $D_{i} \in \mathcal{D}_{G}$ and $g_{i} \in \mathbb{C}^{G}$ is a given function for every $i \in I$, and $f$ is the unknown function. Formally, a system of difference equations is a subset of $\mathcal{D}_{G} \times \mathbb{C}^{G}$. The function $f$ is a solution to the system $S$ if $D f=g$ for every $(D, g) \in S$.

It was proved in [4, Theorem 2.1] that a system $S$ is solvable if and only if it is noncontradictory in the following sense: whenever $\left(D_{i}, g_{i}\right) \in S, A_{i} \in \mathcal{D}_{G}(i=$ $1, \ldots, n)$ and $\sum_{i=1}^{n} A_{i} D_{i}=0$, then $\sum_{i=1}^{n} A_{i} g_{i}=0$. This implies that a system $S$ is solvable if and only if every finite subsystem of $S$ is solvable. However, if we look for solutions belonging to a given class of functions, then the analogous statement is no longer true. For example, there exists a system $S$ on $\mathbb{R}$ such that every finite subsystem of $S$ has a solution which is a trigonometric polynomial, but $S$ has no such solution; moreover, $S$ has no measurable solutions; see [4, Theorem 4.4].

This phenomenon motivates the following definition. Let $\mathcal{F} \subset \mathbb{C}^{G}$ be a class of functions. The solvability cardinal $\operatorname{sc}(\mathcal{F})$ of $\mathcal{F}$ is the smallest cardinal number $\kappa$ with the following property: whenever $S$ is a system of difference equations on $G$ such that each subsystem of $S$ of cardinality less than $\kappa$ has a solution in $\mathcal{F}$, then $S$ itself has a solution in $\mathcal{F}$.

The solvability cardinals of several function classes defined on $\mathbb{R}$ were determined in [1]. As it turned out, the behaviour of $\operatorname{sc}(\mathcal{F})$ is rather erratic. For example, sc $(\mathbb{C}[x])=3$, but sc $(\mathcal{T P})=\omega_{1}$, where $\mathcal{T} \mathcal{P}$ is the set of trigonometric polynomials; $\operatorname{sc}\left(\mathbb{C}^{\mathbb{R}}\right)=\omega$, but $\operatorname{sc}(\mathcal{D F})=\left(2^{\omega}\right)^{+}$, where $\mathcal{D} \mathcal{F}$ is the set of functions having the Darboux property. As for the class $B_{\alpha}$ of Baire $\alpha$ functions defined on $\mathbb{R}$ it was shown in [2] that for every $2 \leq \alpha<\omega_{1}$ we have $\operatorname{sc}\left(B_{\alpha}\right) \geq \omega_{2}$ and, under the continuum hypothesis, we have $\operatorname{sc}\left(B_{\alpha}\right)=\omega_{2}$. The value of $\mathrm{sc}\left(B_{1}\right)$ is not known.

In this paper our main concern is to determine or to estimate the solvability cardinal of the class of polynomial functions defined on $\mathbb{R}^{n}$, on normed linear spaces and, in general, on topological Abelian groups.

The ring of complex valued polynomials of $n$ variables is denoted bybreak $\mathbb{C}\left[x_{1}, \ldots, x_{n}\right]$. Let $G$ be a topological Abelian group. A function $f: G \rightarrow \mathbb{C}$ is called a (complex valued) polynomial, if there is an $n$ such that $f$ can be represented in the form $P\left(a_{1}, \ldots, a_{n}\right)$, where $P \in \mathbb{C}\left[x_{1}, \ldots, x_{n}\right]$ and $a_{1}, \ldots, a_{n}$ are continuous homomorphisms from $G$ into $\mathbb{C}$. We denote by $\mathcal{P}_{G}$ the set of complex valued polynomials defined on $G$. If $G=\mathbb{R}^{k}$, then the continuous 
homomorphisms from $G$ into $\mathbb{C}$ are the linear functions, and thus $\mathcal{P}_{\mathbb{R}^{k}}$ coincides with $\mathbb{C}\left[x_{1}, \ldots, x_{k}\right]$.

As we mentioned above, $\mathrm{sc}\left(\mathcal{P}_{\mathbb{R}}\right)=3$. We prove that if $2 \leq k<\infty$, then $\operatorname{sc}\left(\mathcal{P}_{\mathbb{R}^{k}}\right)=\omega$. On the other hand, if $X$ is a normed linear space of infinite dimension, then $\operatorname{sc}\left(\mathcal{P}_{X}\right)=\omega_{1}$ (see Theorem 5.1).

In general, the value of $\operatorname{sc}\left(\mathcal{P}_{G}\right)$ can be arbitrarily large. Let $r_{0}(G)$ denote the torsion free rank of the Abelian group $G$. That is, let $r_{0}(G)$ denote the cardinality of a maximal independent system of elements of $G$ of infinite order. In Theorem 3.3 we show that every Abelian group $G$ can be made into a topological group in which sc $\left(\mathcal{P}_{G}\right) \geq \kappa^{+}$, where $\kappa=r_{0}(G)$.

We also show that if $G$ is discrete, then sc $\left(\mathcal{P}_{G}\right)=3$ if $r_{0}(G) \leq 1$, sc $\left(\mathcal{P}_{G}\right)=$ $\omega$ if $2 \leq r_{0}(G)<\infty$, and sc $\left(\mathcal{P}_{G}\right) \geq \omega_{1}$ if $r_{0}(G)$ is infinite (see Theorem 6.1). It is not clear whether $\operatorname{sc}\left(\mathcal{P}_{G}\right)=\omega_{1}$ is true for every discrete Abelian group of infinite rank. In Theorem 6.3 we prove that this is true for the class of generalized polynomials defined as follows.

Let $G$ be an Abelian group, and let $h \in G$. The difference operator $\Delta_{h}$ is defined by $\Delta_{h} f(x)=f(x+h)-f(x)$ for every $f: G \rightarrow \mathbb{C}$ and $x \in G$. If $G$ is a topological Abelian group, then we say that a continuous function $f: G \rightarrow \mathbb{C}$ is a generalized polynomial, if there is an $n \geq 0$ such that $\Delta_{h_{1}} \ldots \Delta_{h_{n+1}} f=0$ for every $h_{1}, \ldots, h_{n+1} \in G$. The smallest $n$ with this property is the degree of $f$, denoted by $\operatorname{deg} f$. The degree of the identically zero function is -1 by definition. We denote by $\mathcal{G} \mathcal{P}_{G}$ the set of generalized polynomials defined on $G$.

It is easy to see that $\mathcal{P}_{G} \subset \mathcal{G} \mathcal{P}_{G}$ for every topological Abelian group $G$. If there is a dense subgroup $H$ of $G$ such that $r_{0}(H)<\infty$, then we have $\mathcal{P}_{G}=\mathcal{G} \mathcal{P}_{G}$. (See, e.g. [6, Theorem 9].)

The investigation of the solvability of systems of difference equations was partly motivated by some lifting type questions concerning projections of function classes commuting with translations. For example, it was proved in [4] that if $\Phi$ is a linear map from $\mathbb{C}^{\mathbb{R}}$ to $\mathbb{C}[x]$, then $\Phi$ cannot commute with all translations unless $\Phi$ is identically zero. More precisely, if there is a polynomial $p$ such that $\Phi(p) \neq 0$, then the group

$$
G_{\Phi}=\left\{a \in \mathbb{R}: \Phi\left(T_{a} f\right)=T_{a} \Phi(f) \text { for every } f \in \mathbb{C}^{\mathbb{R}}\right\}
$$

is countable, moreover, its elements are pairwise commensurable. On the other hand, it was shown in [4, Theorem 5.3] that there exists a projection $\Phi$ from $\mathbb{C}^{\mathbb{R}}$ onto $\mathbb{C}[x]$ such that $G_{\Phi}=\mathbb{Q}$. In the last section we generalize this result by showing that there exists a projection from $\mathbb{C}^{\mathbb{R}^{n}}$ onto $\mathbb{C}\left[x_{1}, \ldots, x_{n}\right]$ commuting with translations by vectors having rational coordinates (Theorem 7.4).

The proof of Theorem 7.4 is based on the following result: if $G$ is a discrete Abelian group with $r_{0}(G)<\infty$, and if $S$ is a noncontradictory system of difference equations on $G$ such that for every $(D, g) \in S$ the function $g$ is a polynomial, then $S$ has a polynomial solution (Theorem 6.4). 
We use the following notation. If $G$ is a group and $F \subset G$ is a subset, then we denote by $\langle F\rangle$ the subgroup of $G$ generated by $F$. If $F=\left\{x_{1}, x_{2}, \ldots\right\}$, then we may write $\left\langle x_{1}, x_{2}, \ldots\right\rangle$ instead of $\left\langle\left\{x_{1}, x_{2}, \ldots\right\}\right\rangle$.

The translation operator $T_{h}$ is defined by $T_{h} f(x)=f(x+h)(x \in G)$ for every $h \in G$ and $f: G \rightarrow \mathbb{C}$. Clearly, every difference operator on $G$ can be represented in the form $\sum_{i=1}^{n} c_{i} \cdot T_{b_{i}}$, where $c_{1}, \ldots, c_{n} \in \mathbb{C}$ and $b_{1}, \ldots, b_{n} \in G$. It is easy to check that this representation is unique (apart from the order of terms) if we require $c_{1}, \ldots, c_{n} \neq 0$ and $b_{i} \neq b_{j}(1 \leq i<j \leq n)$.

If $D \in \mathcal{D}_{G}$ and $D=\sum_{j=1}^{n} a_{j} T_{b_{j}}$, where $a_{1}, \ldots, a_{n}$ are nonzero complex numbers and $b_{1}, \ldots, b_{n}$ are distinct elements of $G$, then we put $\operatorname{supp} D=$ $\left\{b_{1}, \ldots, b_{n}\right\}$.

If $(X, \mathcal{T})$ is a topological space, then $C(X)$ denotes the set of continuous functions $f: X \rightarrow \mathbb{C}$.

We denote by $w(X)$ the weight of the topological space $(X, \mathcal{T})$. That is, $w(X)$ is the smallest cardinal $\kappa$ such that $\mathcal{T}$ has a basis of cardinality $\kappa$.

The character of the topological space $(X, \mathcal{T})$ is the smallest cardinal $\lambda$ such that each point of $X$ has a local open basis of cardinality $\lambda$. The character of $(X, \mathcal{T})$ is denoted by $\chi(X)$.

The cardinality of the set $A$ is denoted by $|A|$.

\section{Preliminary results on modules of polynomials}

Let $G$ be an Abelian group. It is easy to see that $\mathcal{D}_{G}$ is an algebra under addition, composition as multiplication, and multiplication with complex numbers. Clearly, $\mathcal{D}_{G}$ is isomorphic to the group ring $\mathbb{C}[G]$. The identity map $T_{0}$ is a unit in $\mathcal{D}_{G}$.

Let $G$ be a topological Abelian group. Then the set $\mathcal{P}_{G}$ of polynomials is invariant under translations. Indeed, if $f=P\left(a_{1}, \ldots, a_{n}\right)$, where $P \in$ $\mathbb{C}\left[x_{1}, \ldots, x_{n}\right]$ and $a_{1}, \ldots, a_{n}$ are continuous additive functions, then

$$
f(x+h)=P\left(a_{1}(x)+a_{1}(h), \ldots, a_{n}(x)+a_{n}(h)\right)
$$

for every $x, h \in G$. Putting $b=\left(a_{1}(h), \ldots, a_{n}(h)\right) \in \mathbb{C}^{n}$ we obtain $T_{h} f=$ $Q\left(a_{1}, \ldots, a_{n}\right)$, where $Q=T_{b} P \in \mathbb{C}\left[x_{1}, \ldots, x_{n}\right]$. Thus $T_{h} f \in \mathcal{P}_{G}$.

Since every difference operator is the linear combination of translations, it follows that $D f \in \mathcal{P}_{G}$ for every $D \in \mathcal{D}_{G}$ and $f \in \mathcal{P}_{G}$. In other words, $\mathcal{P}_{G}$ is a module over the algebra $\mathcal{D}_{G}$. The submodules of $\mathcal{P}_{G}$ are the translation invariant linear subspaces of $\mathcal{P}_{G} \cdot{ }^{1} \mathrm{~A}$ set $A \subset \mathcal{P}_{G}$ is an affine submodule, if $A=M+p$, where $M$ is a submodule of $\mathcal{P}_{G}$ and $p \in \mathcal{P}_{G}$. Submodules and affine submodules are always supposed to be nonempty. Note that if $A$ is

\footnotetext{
${ }^{1}$ In [7] submodules of $\mathbb{C}\left[x_{1}, \ldots, x_{n}\right]$ were defined differently, but $[7$, Lemma 7$]$ shows that the two definitions are equivalent.
} 
an affine submodule and $A=M+p$, where $M$ is a submodule of $\mathcal{P}_{G}$, then $A=M+q$ for every $q \in A$.

Lemma 2.1. Let $S$ be a system of difference equations on a topological Abelian group $G$. If $S$ has a polynomial solution, then the set of all polynomial solutions of $S$ is an affine submodule of $\mathcal{P}_{G}$.

Proof. Let $S=\left\{\left(D_{i}, g_{i}\right): i \in I\right\}$, and let $M=\left\{f \in \mathcal{P}_{G}: D_{i} f=0(i \in I)\right\}$. It is clear that $M$ is a linear subspace of $\mathcal{P}_{G}$. If $h \in G$ and $f \in M$, then $T_{h} f \in$ $M$, since $D_{i} T_{h} f=T_{h} D_{i} f=0$ for every $i \in I$. Therefore, $M$ is translation invariant, hence a submodule of $\mathcal{P}_{G}$.

Let $p \in \mathcal{P}_{G}$ be a solution of $S$. It is clear that the set $A$ of polynomial solutions of $S$ equals $\{p+f: f \in M\}$. Thus $A$ is an affine submodule of $\mathcal{P}_{G}$.

Our next aim is to prove the following.

Theorem 2.2. Suppose there is a dense subgroup $H$ of $G$ such that $r_{0}(H)<\infty$. Then the system of affine submodules of $\mathcal{P}_{G}$ has the minimal condition. That is, if $A_{1} \supset A_{2} \supset \ldots$ is a descending sequence of affine submodules of $\mathcal{P}_{G}$, then there is an $N$ such that $A_{i}=A_{N}$ for every $i \geq N$.

Lemma 2.3. Let $H$ be a subgroup of the topological Abelian group $G$, and let $\bar{H}$ denote the set of elements $x \in G$ such that $n x \in H$ for some nonzero integer $n$. Suppose that $\bar{H}$ is dense in $G$. Then, if a polynomial vanishes on $H$, then it vanishes on $G$.

Proof. Suppose the polynomial $p$ is zero on $H$. Let $h \in \bar{H}$ be given, and let $n$ be a nonzero integer such that $n h \in H$.

Every additive function on the subgroup $\langle h\rangle$ is of the form $a(k h)=c \cdot k$ $(k \in \mathbb{Z})$ with a suitable $c \in \mathbb{C}$. This implies that there is a polynomial $q \in \mathbb{C}[x]$ such that $p(k h)=q(k)$ for every $k \in \mathbb{Z}$. Thus $q(j n)=p(j n h)=0$ for every $j \in \mathbb{Z}$, since $j n h \in H$ and $p$ vanishes on $H$. Hence $q=0$, and $p(h)=q(1)=0$.

This shows $p=0$ on $\bar{H}$. Since $\bar{H}$ is dense in $G$, it follows by continuity that $p=0$ on $G$.

Lemma 2.4. Let $G$ be a topological Abelian group, and suppose that there is a dense subgroup $H$ of $G$ such that $r_{0}(H)=n<\infty$. Then there is an injective and linear map $p \mapsto \bar{p}$ from $\mathcal{P}_{G}$ into $\mathbb{C}\left[x_{1}, \ldots, x_{n}\right]$ such that if $M$ is a submodule of $\mathcal{P}_{G}$, then $\bar{M}=\{\bar{p}: p \in M\}$ is a submodule of $\mathbb{C}\left[x_{1}, \ldots, x_{n}\right]$.

Proof. Let $h_{1}, \ldots, h_{n} \in H$ be independent elements of infinite order. For every $p \in \mathcal{P}_{G}$ there is a polynomial $\bar{p} \in \mathbb{C}\left[x_{1}, \ldots, x_{n}\right]$ such that

$$
p\left(k_{1} h_{1}+\cdots+k_{n} h_{n}\right)=\bar{p}\left(k_{1}, \ldots, k_{n}\right)
$$

for every $k_{1}, \ldots, k_{n} \in \mathbb{Z}$. Indeed, this is clear if $p$ is additive, and then the general statement follows from the fact that every polynomial on $G$ is of the form $P\left(a_{1}, \ldots, a_{m}\right)$, where $P \in \mathbb{C}\left[x_{1}, \ldots, x_{m}\right]$ and $a_{1}, \ldots, a_{m}$ are additive functions. 
We show that the map $p \mapsto \bar{p}$ is injective. Since it is linear, it is enough to show that if $p \in \mathcal{P}_{G}$ and $\bar{p}=0$, then $p=0$. The condition $\bar{p}=0$ means that $p$ is zero on the subgroup $H_{1}=\left\langle h_{1}, \ldots, h_{n}\right\rangle$. For every $h \in H$ there is an integer $n \neq 0$ such that $n h \in H_{1}$, and thus $p=0$ follows from Lemma 2.3.

Next we show that if $M$ is a submodule of $\mathcal{P}_{G}$, then $\bar{M}=\{\bar{p}: p \in M\}$ is a submodule of $\mathbb{C}\left[x_{1}, \ldots, x_{n}\right]$. It is clear that $\bar{M}$ is a linear subspace of $\mathbb{C}\left[x_{1}, \ldots, x_{n}\right]$. In order to prove that $\bar{M}$ is translation invariant it is enough to show that $\bar{M}$ is invariant under translations by elements of $\mathbb{Z}^{n}$ (see $[7$, Lemma $7])$. If $a=\left(a_{1}, \ldots, a_{n}\right) \in \mathbb{Z}^{n}$, then it follows from (1) that $T_{a} \bar{p}=\bar{q}$, where $q=T_{a_{1} h_{1}+\cdots+a_{n} h_{n}} p \in M$, and thus $T_{a} \bar{p} \in \bar{M}$.

Proof of Theorem 2.2. If $r_{0}(H)=0$, then $H$ is torsion, and every element of $\mathcal{P}_{H}$ is constant. Therefore, by continuity, the same is true for the elements of $\mathcal{P}_{G}$. If $M$ is a submodule of $G$, then either $M=\{0\}$, or $M$ equals the set of all constants. Then every affine submodule consists of either a single constant function or the set of all constants. It is clear that this system has the minimal condition.

Now suppose $0<r_{0}(H)=n<\infty$. Let $A_{1} \supset A_{2} \supset \ldots$ be a sequence of affine submodules of $\mathcal{P}_{G}$, and let $A_{k}=M_{k}+p_{k}$, where $M_{k}$ is a submodule of $\mathcal{P}_{G}$ and $p_{k} \in \mathcal{P}_{G}$ for every $k$.

For every $k$ we have $p_{k+1} \in A_{k+1} \subset A_{k}$, and thus $A_{k}=M_{k}+p_{k+1}$. Since $M_{k+1}+p_{k+1}=A_{k+1} \subset A_{k}=M_{k}+p_{k+1}$, it follows that $M_{k+1} \subset M_{k}$. This is true for every $k$, and thus we obtain $M_{1} \supset M_{2} \supset \ldots$

Then $\overline{M_{1}} \supset \overline{M_{2}} \supset \ldots$ is a descending sequence of affine submodules of $\mathbb{R}\left[x_{1}, \ldots, x_{n}\right]$. By $[7$, Lemma 8$]$, we have $\overline{M_{N}}=\overline{M_{N+1}}=\ldots$ for some $N$. Since the map $p \mapsto \bar{p}$ is injective, it follows that $M_{k}=M_{N}$ for every $k \geq N$.

If $k \geq N$, then we have $A_{k+1}=M_{k+1}+p_{k+1}=M_{k}+p_{k+1}=A_{k}$, and we are done.

\section{General estimates}

Theorem 3.1. For every infinite Abelian group $G$ and for every $\mathcal{F} \subset \mathbb{C}^{G}$ we have $\operatorname{sc}(\mathcal{F}) \leq|G|^{+}$.

Proof. We apply the argument of the proof of [1, Theorem 5.1]. Let an ordering $\prec$ of the elements of $G$ be fixed. If $D \in \mathcal{D}_{G}$ and $D=\sum_{j=1}^{n} a_{j} T_{b_{j}}$, where $a_{1}, \ldots, a_{n}$ are nonzero complex numbers and $b_{1}, \ldots, b_{n}$ are distinct elements of $G$ with $b_{1} \prec \ldots \prec b_{n}$, then we put $\varphi(D)=\left(a_{1}, \ldots, a_{n}\right)$.

Let $\mathcal{F} \subset \mathbb{C}^{G}$ be given. We have to show that if $S$ is a system of difference equations on $G$ such that every subsystem of cardinality $\leq|G|$ has a solution belonging to $\mathcal{F}$, then $S$ itself has such a solution. Put $S_{F}=\{(D, g) \in$ $S$ : supp $D=F\}$ for every finite set $F \subset G$. If $F$ is finite, then the space $\mathbb{C}^{|F|}$ is second countable, hence every subset of $\mathbb{C}^{|F|}$ has a countable and 
dense subset. It follows that we can choose a countable $S_{F}^{\prime} \subset S_{F}$ such that $\left\{\varphi(D):(D, g) \in S_{F}^{\prime}\right\}$ is dense in $\left\{\varphi(D):(D, g) \in S_{F}\right\}$.

Put $S^{\prime}=\bigcup_{F \subset G,|F|<\omega} S_{F}^{\prime}$. Then $\left|S^{\prime}\right| \leq|G|$, and thus $S^{\prime}$ has a solution $f \in \mathcal{F}$ by assumption. We show that $f$ is also a solution of $S$; that is, $D f=g$ for every $(D, g) \in S$.

Suppose $(D, g) \in S$, and let $\operatorname{supp} D=F$. Then there is a sequence $\left(D_{i}, g_{i}\right) \in$ $S_{F}^{\prime}$ such that $\varphi\left(D_{i}\right) \rightarrow \varphi(D)$. It is clear that $D_{i} h \rightarrow D h$ pointwise on $G$ for every $h: G \rightarrow \mathbb{C}$.

The system $S^{\prime} \cup\{(D, g)\}$ is also a subsystem of $S$ of cardinality $\leq|G|$, hence it has a solution $f^{\prime} \in \mathcal{F}$. Then we have

$$
D f=\lim _{i \rightarrow \infty} D_{i} f=\lim _{i \rightarrow \infty} g_{i}=\lim _{i \rightarrow \infty} D_{i} f^{\prime}=D f^{\prime}=g .
$$

Since $(D, g) \in S$ was arbitrary, it follows that $f$ is a solution of $S$.

Theorem 3.2. Let $G$ be an Abelian topological group, and put $\kappa=\min (|G|, w(G))$. If $\kappa \geq \omega$, then $\operatorname{sc}(\mathcal{F}) \leq \kappa^{+}$for every $\mathcal{F} \subset C(G)$.

Proof. Let $\mathcal{F} \subset C(G)$ be given. By Theorem 3.1 it is enough to prove

$$
\operatorname{sc}(\mathcal{F}) \leq w(G)^{+} .
$$

We have to show that if $S$ is a system of difference equations on $G$ such that every subsystem of cardinality $\leq w(G)$ has a solution belonging to $\mathcal{F}$, then $S$ itself has such a solution. We fix an ordering $\prec$ of the elements of $G$. If $D \in \mathcal{D}_{G}$ and $D=\sum_{j=1}^{n} a_{j} T_{b_{j}}$, where $a_{1}, \ldots, a_{n}$ are nonzero complex numbers and $b_{1}, \ldots, b_{n}$ are distinct elements of $G$ with $b_{1} \prec \ldots \prec b_{n}$, then we put

$$
\psi(D)=\left(a_{1}, \ldots, a_{n}, b_{1}, \ldots, b_{n}\right) \in \mathbb{C}^{n} \times G^{n} .
$$

Put $S_{n}=\{(D, g) \in S:|\operatorname{supp} D|=n\}$. Since $w(G) \geq \omega$ by assumption, the weight of the product space $\mathbb{C}^{n} \times G^{n}$ equals $w(G)$, hence the weight of each of its subspaces is at most $w(G)$. Therefore, every subset of $\mathbb{C}^{n} \times G^{n}$ has a dense subset of cardinality $\leq w(G)$. It follows that we can choose a subset $S_{n}^{\prime} \subset S_{n}$ such that $\left|S_{n}^{\prime}\right| \leq w(G)$ and $\left\{\psi(D):(D, g) \in S_{n}^{\prime}\right\}$ is dense in $\{\psi(D):(D, g) \in$ $\left.S_{n}\right\}$.

Put $S^{\prime}=\bigcup_{n=1}^{\infty} S_{n}^{\prime}$. Since $\left|S^{\prime}\right| \leq w(G)$, it has a solution $f \in \mathcal{F}$ by assumption. We show that $f$ is also a solution of $S$; that is, $D f=g$ for every $(D, g) \in S$. Fix $(D, g) \in S$ and $x \in G$. Since $\mathcal{F} \subset C(G), f$ is continuous.

Suppose $|\operatorname{supp} D|=n$, and let $\varepsilon>0$ be given. Then there is a neighbourhood $U$ of $\psi(D)$ in $\mathbb{C}^{n} \times G^{n}$ such that $\left|D^{\prime} f(x)-D f(x)\right|<\varepsilon$ for every $D^{\prime} \in \mathcal{D}_{G}$ with $\left|\operatorname{supp} D^{\prime}\right|=n$ and $\psi\left(D^{\prime}\right) \in U$.

The system $S^{\prime} \cup\{(D, g)\}$ is also a subsystem of $S$ of cardinality $\leq w(G)$, hence it has a solution $f^{\prime} \in \mathcal{F}$. Since $f^{\prime}$ is continuous, it follows that there is a neighbourhood $U^{\prime}$ of $\psi(D)$ in $\mathbb{C}^{n} \times G^{n}$ such that $\left|D^{\prime} f^{\prime}(x)-D f^{\prime}(x)\right|<\varepsilon$ for every $D^{\prime} \in \mathcal{D}_{G}$ with $\left|\operatorname{supp} D^{\prime}\right|=n$ and $\psi\left(D^{\prime}\right) \in U^{\prime}$. By the choice of $S_{n}^{\prime}$, we can choose a pair $\left(D^{\prime}, g^{\prime}\right) \in S_{n}^{\prime}$ such that $\psi\left(D^{\prime}\right) \in U \cap U^{\prime}$. Then we have 
$\left|D^{\prime} f(x)-D f(x)\right|<\varepsilon$ and $\left|D^{\prime} f^{\prime}(x)-D f^{\prime}(x)\right|<\varepsilon$. Now $D^{\prime} f=D^{\prime} f^{\prime}=g^{\prime}$ and $D f^{\prime}=g$, thus $|D f(x)-g(x)|<2 \varepsilon$. Since $\varepsilon$ was arbitrary, we obtain $D f(x)=g(x)$.

The next theorem shows that the estimate given by Theorem 3.2 cannot be improved; not even for the class of polynomials in topological Abelian groups.

Theorem 3.3. Let $G$ be an Abelian group with $r_{0}(G)=\kappa \geq \omega$. Then there exists a topology that makes $G$ a completely regular topological group such that $\operatorname{sc}(\mathcal{F}) \geq \kappa^{+}$for every class of functions such that $\mathcal{P}_{G} \subset \mathcal{F} \subset C(G)$.

Proof. Let $B$ be a maximal system of independent elements of $G$ of infinite order. Then we have $|B|=\kappa$. We put $H=\langle B\rangle$. Then every element of $H$ can be written uniquely as $x=\sum_{b \in B} \lambda(x, b) b$, where $\lambda(x, b) \in \mathbb{Z}$ for every $b \in B$, and the set $\{b \in B: \lambda(x, b) \neq 0\}$ is finite.

Every additive function $f: H \rightarrow \mathbb{C}$ can be extended uniquely to $G$ as an additive function. Indeed, the maximality of the set $B$ implies that for every $x \in G$ there is a nonzero integer $n_{x}$ such that $n_{x} \cdot x \in B$. We define $f(x)=f\left(n_{x} \cdot x\right) / n_{x}$ for every $x \in G$. It is easy to see that this definition is independent of the choice of the numbers $n_{x}$, and defines an additive extension of $f$ to $G$.

For every subset $C \subset B$ we denote by $N_{C}$ the set $\left\{x \in H: \sum_{b \in C} \lambda(x, b)=\right.$ $0\}$. Clearly, $N_{C}$ is a subgroup of $G$.

Let $\mathcal{N}$ denote the family of subgroups $N_{C}$ with $C \subset B$ and $|C|<\kappa$. Let the open basis of $\mathcal{T}$ be the family of sets $N+x$, where $N$ runs through the subgroups $N \in \mathcal{N}$ and $x$ runs through the elements of $G$. Then $\mathcal{T}$ makes $G$ a topological group (see [3, (4.21)]). Clearly, each $N \in \mathcal{N}$ is a clopen subgroup. For every $x \in H$ we have $x \in N_{F}+x \subset H$, where $F=\{b \in B: x(b) \neq 0\}$. This shows that $H$ is also an open subgroup of $G$. Consequently, $H$ is clopen as well.

We show that $G$ is Hausdorff. Let $x, y \in G$ be distinct. If $y-x \notin H$, then $H+x$ and $H+y$ are disjoint neighbourhoods of $x$ and $y$. If $y-x \in H$, then $z=y-x \neq 0$, and thus there is a $b \in B$ such that $\lambda(z, b) \neq 0$. Then $z \notin N_{\{b\}}, y \notin N_{\{b\}}+x$, and thus $N_{\{b\}}+x$ and its complement are disjoint neighbourhoods of $x$ and of $y$. This proves that $G$ is Hausdorff. Since $G$ is a topological group, it follows that $G$ is completely regular.

Let $\mathcal{F}$ be a family of functions with $\mathcal{P}_{G} \subset \mathcal{F} \subset C(G)$. We prove that $\operatorname{sc}(\mathcal{F}) \geq \kappa^{+}$.

We define $a(x)=\sum_{b \in B} \lambda(x, b)(x \in H)$. Then $a$ is an additive function defined on $H$. We also denote by $a$ the unique additive extension of $a$ to $G$. Note that $\Delta_{b} a(x)=a(x+b)-a(x)=a(b)=1$ for every $x \in G$ and $b \in B$.

First we show that $a$ is not continuous at 0 . Suppose it is. Then there is an open set $U$ such that $0 \in U$ and $|a(x)|<1$ for every $x \in U$. By the definition of $\mathcal{T}$, we have $0 \in\left(N_{C_{1}}+x_{1}\right) \cap \ldots \cap\left(N_{C_{k}}+x_{k}\right) \subset U$, where $C_{1}, \ldots, C_{k}$ are 
subsets of $B$ of cardinality $<\kappa$, and $x_{1}, \ldots, x_{k} \in G$. Now $0 \in N_{C_{i}}+x_{i}$ implies $x_{i} \in N_{C_{i}}$, and thus $N_{C_{1}} \cap \ldots \cap N_{C_{k}} \subset U$.

Since $\left|C_{1} \cup \cdots \cup C_{k}\right|<\kappa$, we can choose an element $b_{0} \in B \backslash\left(C_{1} \cup \cdots \cup C_{k}\right)$. Then $b_{0} \in N_{C_{1}} \cap \ldots \cap N_{C_{k}}$, and thus $b_{0} \in U$. However, $a\left(b_{0}\right)=1$, which contradicts the choice of $U$. Therefore, $a$ is not continuous at 0 .

Let $S=\left\{\left(\Delta_{b}, 1\right): b \in B\right\}$. If $f$ is a solution of $S$, then $\Delta_{b} f=1=\Delta_{b} a$ for every $b \in B$. Then $\Delta_{b}(f-a)=0$ for every $b \in B$. Now, it is easy to see that the set $\left\{h \in G: \Delta_{h}(f-a)=0\right\}$ is a subgroup of $G$ (see [5, Lemma 15]). Thus we have $\Delta_{h}(f-a)=0$ for every $h \in H$. Therefore, $f-a$ is constant on $H$. Since $H$ is a neighbourhood of 0 and $a$ is not continuous at 0 , it follows that $f$ is not continuous at 0 either. Then, by $\mathcal{F} \subset C(G)$ we find that $S$ does not have a solution belonging to $\mathcal{F}$.

Let $S^{\prime}$ be a subsystem of $S$ of cardinality $<\kappa$. Then $S^{\prime}=\left\{\left(\Delta_{b}, 1\right): b \in A\right\}$, where $A \subset B$ and $|A|<\kappa$. We prove that $S^{\prime}$ has a polynomial solution. Let $f(x)=\sum_{b \in A} \lambda(x, b)$ for every $x \in H$; then $f$ is additive on $H$. We denote also by $f$ the unique additive extension of $f$ to $G$. Then $\Delta_{b} f(x)=f(x+b)-f(x)=$ $f(b)=1$ for every $b \in A$, and thus $f$ is a solution of $S^{\prime}$. Since $f(x)=0$ for every $x \in N_{A}$ and $N_{A}$ is a neighbourhood of 0 , it follows that $f$ is continuous at 0 . Now $f$ is additive, hence $f$ is continuous everywhere, and thus $f$ is a polynomial.

We proved that every subsystem of $S$ of cardinality $<\kappa$ has a polynomial solution, but $S$ itself does not have a continuous solution. Since $\mathcal{P}_{G} \subset \mathcal{F} \subset$ $C(G)$, it follows that $\operatorname{sc}(\mathcal{F}) \geq \kappa^{+}$.

\section{Estimates of $\operatorname{sc}\left(\mathcal{P}_{G}\right)$}

Theorem 4.1. Let $G$ be a topological Abelian group such that every neighbourhood of 0 generates $G$. If $\kappa=\min (|G|, w(G), \chi(G)) \geq \omega$, then $\operatorname{sc}\left(\mathcal{P}_{G}\right) \leq \kappa^{+}$.

Proof. By Theorem 3.2 it is enough to show that $\operatorname{sc}\left(\mathcal{P}_{G}\right) \leq \chi(G)^{+}$.

Every polynomial has the form $P\left(a_{1}, \ldots, a_{n}\right)$, where $P \in \mathbb{C}\left[x_{1}, \ldots, x_{n}\right]$ and $a_{1}, \ldots, a_{n}$ are continuous additive functions on $G$. Let $\mathcal{P}_{N}$ denote the set of polynomials $P \in \mathbb{C}\left[x_{1}, \ldots, x_{N}\right]$ such that $\operatorname{deg} P \leq N$, and the absolute value of each coefficient of $P$ is at most $N$.

Let $\left\{U_{\alpha}: \alpha<\chi(G)\right\}$ be a local base of 0 . For every positive integer $N$ and $\alpha<\chi(G)$ we say that a function $f: G \rightarrow \mathbb{C}$ is an $(N, \alpha)$-polynomial, if $f=P\left(a_{1}, \ldots, a_{N}\right)$, where $P \in \mathcal{P}_{N}$, and $a_{1}, \ldots, a_{N}: G \rightarrow \mathbb{C}$ are additive functions such that $\left|a_{i}(x)\right| \leq 1$ for every $x \in U_{\alpha}$ and $i=1, \ldots, N$. By an $(N, \alpha)$-solution of a system of difference equations we mean a solution which is an $(N, \alpha)$-polynomial.

Let $S$ be a system of difference equations on $G$ such that every subsystem of cardinality $\leq \chi(G)$ has a polynomial solution. We have to show that $S$ itself has a polynomial solution. 
We prove that there is a positive integer $N$ and there is an $\alpha<\chi(G)$ such that every finite subsystem of $S$ has an $(N, \alpha)$-solution.

Suppose there is no such pair $(N, \alpha)$. Then for every pair $(N, \alpha)$ with $N \in$ $\mathbb{N}^{+}$and $\alpha<\chi(G)$ there is a finite subsystem $S_{(N, \alpha)} \subset S$ having no $(N, \alpha)$ solution. Let $S^{\prime}=\bigcup_{N=1}^{\infty} \bigcup_{\alpha<\chi(G)} S_{(N, \alpha)}$. Then $\left|S^{\prime}\right| \leq \chi(G)$, hence $S^{\prime}$ has a polynomial solution $f$. Suppose $f=P\left(a_{1}, \ldots, a_{n}\right)$, where $P \in \mathbb{C}\left[x_{1}, \ldots, x_{n}\right]$ and $a_{1}, \ldots, a_{n}$ are continuous additive functions. Clearly, there is an $N$ such that $N \geq n$ and $P \in \mathcal{P}_{N}$. Since $a_{1}, \ldots, a_{n}$ are continuous at 0 , there is an $\alpha<\chi(G)$ such that $\left|a_{i}(x)\right| \leq 1$ for every $x \in U_{\alpha}$ and $i=1, \ldots, N$. Then $f$ is an $(N, \alpha)$-polynomial. Thus $S^{\prime}$ and, consequently, every subsystem of $S^{\prime}$ has an $(N, \alpha)$-solution. In particular, $S_{(N, \alpha)}$ has an $(N, \alpha)$-solution, which is impossible.

We fix a positive integer $N$ and an $\alpha<\chi(G)$ such that every finite subsystem of $S$ has an $(N, \alpha)$-solution.

Since, by assumption, $U_{\alpha}$ generates $G$, every $x \in G$ belongs to $n(x) \cdot U_{\alpha}$ with a smallest positive integer $n(x)$ depending on $x .^{2}$ Thus $n(x)=1$ for every $x \in U_{\alpha}$.

Put $D_{r}=\{z \in \mathbb{C}:|z| \leq r\}$ for every $r>0$. Let $Y$ denote the set of maps $u: G \rightarrow \mathbb{C}$ such that $u(x) \in D_{n(x)}$ for every $x \in G$. Since $D_{r}$ is a compact subset of $\mathbb{C}$ for every $r>0$, it follows that $Y$, endowed with the product topology, is a compact topological space. Let $A$ denote the set of additive maps $u \in Y$. It is easy to check that $A$ is a closed subset of $Y$ and thus $A$, as a subspace of $Y$, is compact. It is clear that $A$ contains every additive function $a$ such that $|a(x)| \leq 1\left(x \in U_{\alpha}\right)$.

Note that every $a \in A$ is continuous. Indeed, if $\varepsilon>0$ is given, then let $k>1 / \varepsilon$, and let $V$ be a neighbourhood of 0 such that $k \cdot V \subset U_{\alpha}$. If $a \in A$ and $x \in V$, then $k x \in U_{\alpha},|a(k x)| \leq 1, k \cdot|a(x)|=|a(k x)| \leq 1$ and $|a(x)|<1 / k<\varepsilon$. Thus $a$ is continuous at 0 and then, being additive, it is continuous everywhere.

Let $M$ denote the number of sequences $\left(i_{1}, \ldots, i_{N}\right)$ such that $i_{1}, \ldots, i_{N}$ are nonnegative integers with $i_{1}+\cdots+i_{N} \leq N$. Then $M$ is the number of terms in any polynomial $P \in \mathcal{P}_{N}$. Let $\phi(P)$ be the sequence of coefficients of $P$ listed in a fixed order. Then $\phi$ is a bijective map from $\mathcal{P}_{N}$ onto $\left(D_{N}\right)^{M}$. The set $\left(D_{N}\right)^{M}$, as a subspace of the product space $\mathbb{C}^{M}$, is compact. Let $P_{N}$ be endowed with topology $\left\{\phi^{-1}(U): U\right.$ is open in $\left.\left(D_{N}\right)^{M}\right\}$. This makes $\mathcal{P}_{N}$ a compact topological space.

We prove that there is a sequence $\left(P, a_{1}, \ldots, a_{N}\right) \in \mathcal{P}_{N} \times A^{N}$ such that $P\left(a_{1}, \ldots, a_{N}\right)$ is a solution of $S$. Suppose this is not true.

If $\left(P, a_{1}, \ldots, a_{N}\right) \in \mathcal{P}_{N} \times A^{N}$ is not such a sequence, then there is a pair $(D, g) \in S$ such that $D P\left(a_{1}, \ldots, a_{N}\right) \neq g$. Let $x \in G$ be such that $D P\left(a_{1}, \ldots, a_{N}\right)(x) \neq g(x)$. It is clear that if we replace $P$ by a polynomial $Q$ which is close enough in the topology of $\mathcal{P}_{N}$ and replace $a_{1}, \ldots, a_{N}$ by

${ }^{2}$ By $n \cdot A$ we mean the set $\left\{a_{1}+\cdots+a_{n}: a_{1}, \ldots, a_{n} \in A\right\}$. 
additive functions $b_{1}, \ldots, b_{N}$ which are close enough in the topology of $A$, then $D Q\left(b_{1}, \ldots, b_{N}\right)(x) \neq g(x)$ will hold. (Note that if $\delta>0$, then $\{b \in$ $A:|b(x)-a(x)|<\delta\}$ is a neighbourhood of $a$ in the topology of $A$.) That is, if $P\left(a_{1}, \ldots, a_{N}\right)$ is not a solution of $S$, then there is a neighbourhood $U$ of $\left(P, a_{1}, \ldots, a_{N}\right)$ in $\mathcal{P}_{N} \times A^{N}$ and there is a pair $(D, g) \in S$ such that $D Q\left(b_{1}, \ldots, b_{N}\right) \neq g$ for every $\left(Q, b_{1}, \ldots, b_{n}\right) \in U$.

Now the product space $\mathcal{P}_{N} \times A^{N}$ is compact, and thus it is covered by finitely many of these neighbourhoods. That is, there are finitely many pairs $\left(D_{i}, g_{i}\right)(i=1, \ldots, s)$ such that for every $\left(P, a_{1}, \ldots, a_{N}\right) \in \mathcal{P}_{N} \times A^{N}$ there is an $i \in\{1, \ldots, s\}$ such that $D_{i} P\left(a_{1}, \ldots, a_{N}\right) \neq g_{i}$. This, however, is impossible. Indeed, $\left\{\left(D_{i}, g_{i}\right): i=1, \ldots, s\right\}$ is a finite subsystem of $S$, and thus it has a solution of the form $P\left(a_{1}, \ldots, a_{N}\right)$, where $\left(P, a_{1}, \ldots, a_{N}\right) \in \mathcal{P}_{N} \times A^{N}$. This contradiction proves that $S$ has a solution of the form $P\left(a_{1}, \ldots, a_{N}\right)$, where $\left(P, a_{1}, \ldots, a_{N}\right) \in \mathcal{P}_{N} \times A^{N}$.

Corollary 4.2. If $G$ is a connected and metrizable Abelian topological group, then $\operatorname{sc}\left(\mathcal{P}_{G}\right) \leq \omega_{1}$.

Proof. It is easy to check that if $G=\{0\}$, then $\operatorname{sc}(\mathcal{F}) \leq 3$ for every $\mathcal{F} \subset \mathbb{C}^{G}$, and thus the statement of the corollary is true.

Suppose $|G| \geq 2$. Since $G$ is connected and metrizable, it follows that $|G|$ and $w(G)$ are both infinite. By metrizability we also have $\chi(G)=\omega$, and then the statement follows from Theorem 4.1.

Theorem 4.3. Suppose there is a dense subgroup $H$ of $G$ such that $r_{0}(H)<\infty$. Then $\operatorname{sc}\left(\mathcal{P}_{G}\right) \leq \omega$.

Proof. We have to show that if every finite subsystem of a system $S$ of difference equations has a polynomial solution, then so has $S$.

Let $S=\left\{\left(D_{i}, p_{i}\right): i \in I\right\}$. For every $J \subset I$ let $A_{J}$ denote the set of polynomial solutions of the system $S_{J}=\left\{\left(D_{i}, p_{i}\right): i \in J\right\}$. If $J$ is finite, then $A_{J}$ is nonempty by assumption, and thus it is an affine submodule of $\mathcal{P}_{G}$ by Lemma 2.1.

The system of affine submodules of $\mathcal{P}_{G}$ has the minimal condition by Theorem 2.2. Since $A_{J_{1}} \subset A_{J_{2}}$ for every $J_{1} \supset J_{2}$, it follows that there is a finite $F \subset I$ such that $A_{F} \subset A_{J}$ for every finite $J \subset I$.

It is clear that every $p \in A_{F}$ is a solution of $S_{J}$ for every finite $J \subset I$. Then $D_{i} p=p_{i}$ for every $i \in I$; that is, $p$ is a polynomial solution of $S$.

Now we turn to a lower estimate of $\operatorname{sc}\left(\mathcal{P}_{G}\right)$.

Theorem 4.4. Let $G$ be a topological Abelian group. If there are linearly independent continuous additive functions $a, b: G \rightarrow \mathbb{C}$, then $\operatorname{sc}\left(\mathcal{P}_{G}\right) \geq \omega$.

Proof. We have to show that for every positive integer $n$ there exists a system $S$ of difference equations on $G$ such that every $n$-element subsystem of $S$ has a polynomial solution, but $S$ does not have a polynomial solution. 
If $f: G \rightarrow \mathbb{C}$ is additive, $h \in G$ and $i \geq 2$ is an integer, then

$$
\begin{aligned}
\Delta_{h} f^{i}(x) & =f(x+h)^{i}-f(x)^{i}=(f(x)+f(h))^{i}-f(x)^{i} \\
& =i \cdot f(x)^{i-1} f(h)+\cdots+f(h)^{i}
\end{aligned}
$$

for every $x \in G$. Therefore, we have

$$
\Delta_{h} f^{i}=i \cdot f(h) \cdot f^{i-1}+P(f),
$$

where $P \in \mathbb{C}[x]$ is a polynomial of degree $\leq i-2$. Using (3) it is easy to prove by induction on $i$ that

$$
\Delta_{h}^{i} f^{i}=i ! \cdot f(h)^{i} \quad(i=1,2, \ldots)
$$

holds for every additive function $f: G \rightarrow \mathbb{C}$.

Let $a, b$ be linearly independent continuous additive functions on $G$. Then there are elements $u, v \in G$ such that the vectors $(a(u), a(v))$ and $(b(u), b(v))$ are linearly independent. Then, for every $k \in \mathbb{C}$ there are numbers $\alpha, \beta \in \mathbb{C}$ such that $A(u)=k$ and $A(v)=1$, where $A=\alpha a+\beta b$. We fix the elements $u$ and $v$ with this property.

Let $n \geq 2$ be fixed. Let $c_{1}, \ldots, c_{n}$ be distinct complex numbers, and put

$$
D_{i}=\prod_{1 \leq j \leq n, j \neq i}\left(\Delta_{u}-c_{j} \Delta_{v}\right) \quad(i=1, \ldots, n) .
$$

First we show that if $f: G \rightarrow \mathbb{C}$ is such that $D_{i} f=0$ for every $i=1, \ldots, n$, then $\Delta_{u}^{n-1} f=0$. We prove this statement by induction on $n$.

If $n=2$, then $\left(\Delta_{u}-c_{2} \Delta_{v}\right) f=0$ and $\left(\Delta_{u}-c_{1} \Delta_{v}\right) f=0$ imply $\left(c_{2}-c_{1}\right) \Delta_{v} f=$ $0, \Delta_{v} f=0$, hence $\Delta_{u} f=0$.

Let $n>2$, and suppose the statement is true for $n-1$. Suppose $D_{i} f=0$ for every $i=1, \ldots, n$, and put $g=\left(\Delta_{u}-c_{n} \Delta_{v}\right) f$. Then

$$
\prod_{1 \leq j \leq n-1, j \neq i}\left(\Delta_{u}-c_{j} \Delta_{v}\right) g=0
$$

for every $i=1, \ldots, n-1$. By the induction hypothesis, this implies $\Delta_{u}^{n-2} g=0$; that is, $\Delta_{u}^{n-2}\left(\Delta_{u}-c_{n} \Delta_{v}\right) f=0$. A similar argument gives $\Delta_{u}^{n-2}\left(\Delta_{u}-c_{1} \Delta_{v}\right) f=$ 0 . Therefore, we obtain $\Delta_{u}^{n-2}\left(c_{n}-c_{1}\right) \Delta_{v} f=0, \Delta_{u}^{n-2} \Delta_{v} f=0$ and $\Delta_{u}^{n-2} \Delta_{u} f=$ 0 , proving the statement.

Now let $n \geq 3$ be fixed, and put

$$
D_{i}=\prod_{1 \leq j \leq n, j \neq i}\left(\Delta_{u}-j \Delta_{v}\right) \quad(i=1, \ldots, n) .
$$

Then, by what we proved above, the system

$$
S=\left\{\left(D_{i}, 0\right): i=1, \ldots, n\right\} \cup\left\{\left(\Delta_{u}^{n-1}, 1\right)\right\}
$$

has no solutions; in particular, it has no polynomial solution. 
We show that whenever $S^{\prime}$ is an $n$-element subsystem of $S, S^{\prime}$ has a polynomial solution. This is clear if $S^{\prime}$ is obtained by removing the equation $\Delta_{u}^{n-1} f=1$ from $S$, since $f=0$ is a solution of $S^{\prime}$ in this case.

Suppose that $S^{\prime}$ is obtained by removing the equation $D_{k} f=0$ from $S$, where $1 \leq k \leq n$. As we noted before, there exists a continuous additive function $\bar{A}$ such that $A(u)=k$ and $A(v)=1$. Then, by (3), we have

$\Delta_{u} A^{n-1}=(n-1) \cdot k \cdot A^{n-2}+P(A) \quad$ and $\quad \Delta_{v} A^{n-1}=(n-1) \cdot A^{n-2}+Q(A)$,

where $P, Q$ are polynomials of degree $\leq n-3$. Therefore, $\left(\Delta_{u}-k \Delta_{v}\right) A^{n-1}=$ $R(A)$, where $R$ is a polynomial of degree $\leq n-3$. In particular, $\left(\Delta_{u}-k \Delta_{v}\right) A^{n-1}$ is a generalized polynomial of degree $\leq n-3$.

Now $D_{i}$ is the product of $n-1$ difference operators of the form $\Delta_{u}-j \cdot \Delta_{v}$. If $i \neq k$, then one of these factors is $\Delta_{u}-k \cdot \Delta_{v}$. Since $\left(\Delta_{u}-k \Delta_{v}\right) A^{n-1}$ is a generalized polynomial of degree $\leq n-3$, and the degree of a generalized polynomial is strictly decreasing if we apply a difference operator $\Delta_{u}-j \cdot \Delta_{v}$ to it, we find that $D_{i} A^{n-1}=0$ for every $i \neq k$.

By (4) we have $\Delta_{u}^{n-1} A^{n-1}=(n-1) ! \cdot k^{n-1}$. Therefore, the function $p=$ $A^{n-1} /\left((n-1) ! \cdot k^{n-1}\right)$ is a solution of $S^{\prime}$. Since $p$ is a polynomial, this proves the theorem.

\section{Normed linear spaces}

In this section our aim is to prove the following theorem.

Theorem 5.1. If $X$ is a normed linear space over $\mathbb{R}$, then

$$
\operatorname{sc}\left(\mathcal{P}_{X}\right)= \begin{cases}3 & \text { if } \operatorname{dim} X=1, \\ \omega & \text { if } 2 \leq \operatorname{dim} X<\infty \\ \omega_{1} & \text { if } \operatorname{dim} X \geq \infty .\end{cases}
$$

Proof. If $\operatorname{dim} X=1$, then $X=\mathbb{R}$. The statement $\operatorname{sc}\left(\mathcal{P}_{\mathbb{R}}\right)=3$ was proved in [4, Theorem 4.5].

If $\operatorname{dim} X=k<\infty$, then $\operatorname{sc}\left(\mathcal{P}_{X}\right) \leq \omega$. Indeed, in this case we may assume that $X=\mathbb{R}^{k}$. Then $\mathbb{Q}^{k}$ is dense in $X$ and $r_{0}\left(\mathbb{Q}^{k}\right)=k$, and we can apply Theorem 4.3.

Suppose $\operatorname{dim} X \geq 2$, and let $u, v$ be linearly independent elements of $X$. The Hahn-Banach theorem implies that there are continuous linear functionals $a, b: X \rightarrow \mathbb{C}$ such that $a(u)=1, a(v)=0, b(u)=0$ and $b(v)=1$. Then $\operatorname{sc}\left(\mathcal{P}_{X}\right) \geq \omega$ follows from Theorem 4.4 .

If $X$ is a metrizable topological linear space, then $X$ is connected, and thus $\operatorname{sc}\left(\mathcal{P}_{X}\right) \leq \omega_{1}$ follows from Corollary 4.2. Therefore, in order to complete the proof of Theorem 5.1 it is enough to prove the following. 
Theorem 5.2. Let $X$ be an infinite dimensional normed linear space over $\mathbb{R}$. Then we have $\operatorname{sc}(\mathcal{F}) \geq \omega_{1}$ for every set of functions such that $\mathcal{P}_{X} \subset \mathcal{F} \subset$ $C(X)$.

Proof. We construct a system $S$ of difference equations on $X$ such that every finite subsystem has a polynomial solution, but $S$ does not have continuous solutions.

Let $B$ be a basis of $X$ as a linear space over $\mathbb{R}$. By assumption, $B$ is infinite. Let $b_{1}, b_{2}, \ldots$ be distinct elements of $B$. By multiplying these elements by suitable nonzero complex numbers we may assume that $\left\|b_{i}\right\| \rightarrow 0$ as $i \rightarrow \infty$.

Every $x \in X$ has a unique representation in the form $\sum_{b \in B} \lambda(x, b) \cdot b$, where $\lambda(x, b) \in \mathbb{R}$, and the set $\{b \in B: \lambda(x, b) \neq 0\}$ is finite. Let $a(x)=\sum_{b \in B} \lambda(b, x)$. Then $a: X \rightarrow \mathbb{R}$ is a linear map. Since $a\left(b_{i}\right)=1$ for every $i=1,2, \ldots$ and $b_{i} \rightarrow 0=a(0)$, it follows that $a$ is not continuous.

Now the restriction of $a$ to every finite dimensional linear subspace $V \subset X$ is continuous, since a finite dimensional linear space has only continuous linear functionals.

Let $S=\left\{\left(\Delta_{h}, a(h)\right): h \in X\right\}$. We show that every finite subsystem of $S$ has a polynomial solution. If $h_{1}, \ldots, h_{n} \in X$, then $a$ is continuous, hence a bounded linear functional on the linear subspace $V$ generated by $h_{1}, \ldots, h_{n}$. By the Hahn-Banach theorem $\left.a\right|_{V}$ can be extended to $X$ as a bounded linear functional $A$. If $h \in V$, then $\Delta_{h} A(x)=A(h)=a(h)$ for every $x \in X$, and thus $A$ is a solution of the system $S^{\prime}=\left\{\left(\Delta_{h_{i}}, a\left(h_{i}\right)\right): i=1, \ldots, n\right\}$. Now $A$ is a continuous additive function, hence a polynomial, showing that $S^{\prime}$ has a polynomial solution.

On the other hand, if $f$ is a solution of $S$, then $\Delta_{h} f=a(h)=\Delta_{h} a$ for every $h \in X$, and thus $f=a+c$, where $c$ is a constant. Since $a$ is not continuous, $f$ is not continuous either, and thus $S$ does not have continuous solutions.

\section{Discrete Abelian groups}

Our first aim is to prove the following.

Theorem 6.1. If $G$ is a discrete Abelian group, then

$$
\operatorname{sc}\left(\mathcal{P}_{G}\right)= \begin{cases}3 & \text { if } r_{0}(G) \leq 1, \\ \omega & \text { if } 2 \leq r_{0}(G)<\infty, \\ \geq \omega_{1} & \text { if } r_{0}(G) \geq \infty .\end{cases}
$$

Proof. If $r_{0}(G)=0$, then $G$ is torsion. Then every additive function on $G$ is constant and, consequently, every polynomial on $G$ is constant. Now the set of constant solutions of a difference equation $D f=g$ is either empty or consists of one single constant function, or consists of all constant functions. From this observation it is easy to prove that $\operatorname{sc}\left(\mathcal{P}_{G}\right)=3$. 
To deal with the case $r_{0}(G)=1$ we need some simple facts on the affine submodules of $\mathbb{C}[x]$. It is easy to see that if $M$ is a submodule of $\mathbb{C}[x]$, then either $M=\mathbb{C}[x]$, or $M=\{p \in \mathbb{C}[x]: \operatorname{deg} p \leq d\}$ for some integer $d \geq-1$. Consequently, if $M_{1}, M_{2}$ are submodules of $\mathbb{C}[x]$, then either $M_{1} \subset M_{2}$ or $M_{2} \subset M_{1}$. Suppose $A_{1}, A_{2}$ are affine submodules of $\mathbb{C}[x]$ such that $A_{1} \cap A_{2} \neq \emptyset$. If $p \in A_{1} \cap A_{2}$, then $A_{i}=p+M_{i}(i=1,2)$, where $M_{1}, M_{2}$ are submodules. Therefore, we have either $A_{1} \subset A_{2}$ or $A_{2} \subset A_{1}$ whenever $A_{1}, A_{2}$ are affine submodules of $\mathbb{C}[x]$ with nonempty intersection. Thus, if $\mathcal{A}$ is a set of affine submodules of $\mathbb{C}[x]$ such that $A_{1} \cap A_{2} \neq \emptyset$ for every $A_{1}, A_{2} \in \mathcal{A}$, then $\mathcal{A}$ has a minimal element.

Now suppose $r_{0}(G)=1$, and let $S$ be a system of difference equations on $G$. For every $(D, g) \in S$ the set of polynomial solutions of $D f=g$ constitutes an affine submodule $A_{D, g}$ of $\mathcal{P}_{G}$. If every two-element subsystem of $S$ has a polynomial solution, then the intersection of any two of these affine submodules $A_{D, g}$ is nonempty. Let $p \mapsto \bar{p}$ be the map from $\mathcal{P}_{g}$ into $\mathbb{C}[x]$ as in Lemma 2.4. Then the intersection of any two of the affine submodules $\bar{A}_{D, g}$ of $\mathbb{C}[x]$ is nonempty. Therefore, among the affine submodules $\bar{A}_{D, g}$ there is a minimal one. If $\bar{A}_{D_{0}, g_{0}}$ is minimal, then $A_{D_{0}, g_{0}}$ is a minimal element of the set $\left\{A_{D, g}:(D, g) \in S\right\}$, and thus every element of $A_{D_{0}, g_{0}}$ is a solution of $S$. This proves sc $\left(\mathcal{P}_{G}\right) \leq 3$. Since $\operatorname{sc}\left(\mathcal{P}_{G}\right) \geq 3$ is obvious, we have $\operatorname{sc}\left(\mathcal{P}_{G}\right)=3$.

If $r_{0}(G)<\infty$, then sc $\left(\mathcal{P}_{G}\right) \leq \omega$ follows from Theorem 4.3. We prove that if $r_{0} \geq 2$, then $\operatorname{sc}\left(\mathcal{P}_{G}\right) \geq \omega$. Let $x_{1}, x_{2}$ be independent elements of $G$ of infinite order. Then every element of the subgroup $H$ generated by $x_{1}, x_{2}$ has a unique representation of the form $k_{1} x_{1}+k_{2} x_{2}$, where $k_{1}, k_{2} \in \mathbb{Z}$. Putting $a(x)=k_{1}$ and $b(x)=k_{2}$ if $x=k_{1} x_{1}+k_{2} x_{2} \in H$, we obtain two additive functions defined on $H$. Extending $a$ and $b$ from $H$ to $G$ as additive functions, we get (continuous) additive functions with $a\left(x_{1}\right)=1, a\left(x_{2}\right)=0, b\left(x_{1}\right)=0, b\left(x_{2}\right)=1$. It is clear that $a$ and $b$ are linearly independent. Therefore, by Theorem 4.4, we have $\operatorname{sc}\left(\mathcal{P}_{G}\right) \geq \omega$.

In order to complete the proof of Theorem 6.1 , it is enough to show that if $r_{0}(G)=\infty$, then $\operatorname{sc}\left(\mathcal{P}_{G}\right) \geq \omega_{1}$. The following theorem states slightly more.

Theorem 6.2. Let $G$ be a discrete Abelian group with $r_{0}(G)=\infty$. Then there is a system $S$ of difference equations on $G$ such that every finite subsystem of $S$ has a polynomial solution, but $S$ does not have a generalized polynomial solution.

Proof. Let $h_{0}, h_{1}, \ldots \in G$ be independent elements of infinite order, and let $D_{0}=\Delta_{h_{0}}$ and $D_{n}=\Delta_{h_{0}}-\Delta_{h_{n}}^{n}(n=1,2, \ldots)$. We put

$$
S=\left\{\left(D_{n}, 0\right): n=1,2, \ldots\right\} \cup\left\{\left(D_{0}, 1\right)\right\} .
$$

Let $H=\left\langle h_{0}, h_{1}, \ldots\right\rangle$. If $x=m_{0} h_{0}+m_{1} h_{1}+\cdots$, where $m_{0}, m_{1}, \ldots$ are integers and $m_{i}=0$ for every $i \geq i_{0}$, then we put $a_{i}(x)=m_{i}(i=0,1, \ldots)$. Then 
$a_{i}$ is an additive function defined on $H$. Each $a_{i}$ can be extended to $G$ as an additive function mapping $G$ into $\mathbb{Q}$. We also denote the extension by $a_{i}$. Note that $\Delta_{h_{i}} a_{j}=0$ if $i \neq j$, and $\Delta_{h_{n}} a_{n}=1$ for every $n=1,2, \ldots$

By (4), we have $\Delta_{h_{n}}^{n} a_{n}^{n}=n$ ! for every $n=1,2, \ldots$ If $p_{N}=a_{0}+\sum_{i=1}^{N} \frac{1}{i !} \cdot a_{i}^{i}$, then $p_{N}$ is a polynomial on $G$, and satisfies $D_{0} p_{N}=\Delta_{h_{0}} p_{N}=1$ and

$$
D_{n} p_{N}=\Delta_{h_{0}} p_{N}-\sum_{i=1}^{N} \frac{1}{i !} \Delta_{h_{n}}^{n} a_{i}^{i}=1-1=0
$$

for every $1 \leq n \leq N$. This shows that every finite subsystem of $S$ has a polynomial solution.

Suppose $S$ has a solution $f$ which is a generalized polynomial. Let $\operatorname{deg} f=d$. Then, for every $n>d$ we have $\Delta_{h_{n}}^{n} f=0$. Thus $\Delta_{h_{0}} f=1$ and

$$
0=D_{n} f=\Delta_{h_{0}} f-\Delta_{h_{n}}^{n} f=1-0=1,
$$

which is impossible.

We do not know whether or not $\operatorname{sc}\left(\mathcal{P}_{G}\right)=\omega_{1}$ holds for every discrete Abelian group $G$ with $r_{0}(G)=\infty$. We prove, however, that this is true for the class of generalized polynomials.

Theorem 6.3. We have $\operatorname{sc}(\mathcal{G P} G)=\omega_{1}$ for every discrete Abelian group $G$ with $r_{0}(G)=\infty$.

Proof. By Theorem 6.2 we have $\operatorname{sc}\left(\mathcal{G P}_{G}\right) \geq \omega_{1}$, so we only have to prove $\operatorname{sc}\left(\mathcal{G} \mathcal{P}_{G}\right) \leq \omega_{1}$. Let $S$ be a system of difference equations on $G$ such that every countable subsystem has a generalized polynomial solution. We have to show that $S$ itself has a generalized polynomial solution.

First we prove that there exists a positive integer $N$ such that every finite subsystem of $S$ has a generalized polynomial solution $f$ with $\operatorname{deg} f<N$. Suppose there is no such $N$. Then for every $N$ there is a finite subsystem $S_{N} \subset S$ having no generalized polynomial solution of degree $<N$. Let $S^{\prime}=$ $\bigcup_{N=1}^{\infty} S_{N}$. Then $\left|S^{\prime}\right| \leq \omega$, thus $S^{\prime}$ has a generalized polynomial solution $f$. Let $N$ be an integer greater than $\operatorname{deg} f$. Since $S_{N} \subset S^{\prime}$, it follows that $f$ is a solution of $S_{N}$ and $\operatorname{deg} f<N$, which contradicts the choice of $S_{N}$.

Fix $N$ such that every finite subsystem of $S$ has a polynomial solution with $\operatorname{deg} f<N$. Every generalized polynomial with $\operatorname{deg} f<N$ satisfies the equation $\Delta_{h_{1}} \ldots \Delta_{h_{N}} f=0$ for every $h_{1}, \ldots, h_{N} \in G$. Therefore, if

$$
\bar{S}=S \cup\left\{\left(\Delta_{h_{1}} \ldots \Delta_{h_{N}}, 0\right): h_{1}, \ldots, h_{N} \in G\right\},
$$

then the system $\bar{S}$ also has the property that each of its finite subsystems has a generalized polynomial solution with $\operatorname{deg} f<N$.

In particular, every finite subsystem of $\bar{S}$ has a solution, hence it is noncontradictory. Then $\bar{S}$ itself is noncontradictory, thus solvable. Let $f$ be a solution of $\bar{S}$. Then $f$ is a solution of $S$, and satisfies $\Delta_{h_{1}} \ldots \Delta_{h_{N}} f=0$ for every $h_{1}, \ldots, h_{N} \in G$. Thus $f$ is a generalized polynomial solution of $S$. 
The following result will be needed in the application discussed in the next section.

Theorem 6.4. Let $G$ be a discrete Abelian group with $r_{0}(G)<\infty$, and let $S$ be a system of difference equations on $G$ such that for every $(D, g) \in S$ the function $g$ is a polynomial. If $S$ is noncontradictory, then $S$ has a polynomial solution.

Proof. The proof will be given in four steps. For every $D_{0} \in \mathcal{D}_{G}$ and $\mathcal{A} \subset \mathcal{D}_{G}$ we denote by $S\left(D_{0}, \mathcal{A}\right)$ the system of equations $\left\{\left(D_{0}, 1\right)\right\} \cup\{(D, 0): D \in \mathcal{A}\}$. I. First we assume $G=\mathbb{Z}^{n}$, and put $\mathcal{D}=\mathcal{D}_{\mathbb{Z}^{n}}$. We show that $\mathcal{D}$, as a commutative ring is Noether. Indeed, let $\mathcal{D}_{\geq 0}$ be the set of difference operators of the form $D=\sum_{i=1}^{k} a_{i} T_{b_{i}}$, where $a_{1}, \ldots, a_{k} \in \mathbb{C}$ and the vectors $b_{1}, \ldots, b_{k} \in \mathbb{Z}^{n}$ have nonnegative coordinates. Then $\mathcal{D}_{\geq 0}$ is a subalgebra of $\mathcal{D}$, and for every $D \in \mathcal{D}$ we have $T_{b} D \in \mathcal{D}_{\geq 0}$ for some $b \in \mathbb{Z}^{n}$. This implies that if $J$ is an ideal of $\mathcal{D}$, then $J \cap \mathcal{D}_{\geq 0}$ is an ideal of $\mathcal{D}_{\geq 0}$, and every basis of $J \cap \mathcal{D}_{\geq 0}$ is also a basis of $J$. Thus it is enough to show that $\mathcal{D}_{\geq 0}$ is Noether. This follows from the fact that $\mathcal{D}_{\geq 0}$ is isomorphic to $\mathbb{C}\left[x_{1}, \ldots, x_{n}\right]$. The isomorphism is $\phi(D)=\sum_{i=1}^{k} a_{i} x^{b_{i}}$, where $D=\sum_{i=1}^{k} a_{i} T_{b_{i}}$ and $x^{b}$ denotes $x_{1}^{k_{1}} \cdots x_{n}^{k_{n}}$, where $b=\left(k_{1}, \ldots, k_{n}\right)$.

First we prove that if $D_{0} \in \mathcal{D}, \mathcal{A} \subset \mathcal{D}$ and $S\left(D_{0}, \mathcal{A}\right)$ is noncontradictory, then $S\left(D_{0}, \mathcal{A}\right)$ has a polynomial solution.

Let $J$ denote the ideal of $\mathcal{D}$ generated by $\mathcal{A}$. It is clear that if $S\left(D_{0}, \mathcal{A}\right)$ is solvable then so is $S\left(D_{0}, J\right)$, and they have the same solutions. Therefore, we may assume that $\mathcal{A}$ is an ideal.

For every ideal $J$, the fact that $S\left(D_{0}, J\right)$ is noncontradictory means that whenever $E_{0}, \ldots, E_{n} \in \mathcal{D}, D_{1}, \ldots, D_{n} \in J$ and $E_{0} D_{0}+\sum_{i=1}^{n} E_{i} D_{i}=0$, we have $E_{o} 1=0$. (In this context 1 denotes the identically 1 function on $G$.) Since $J$ is an ideal, this is equivalent to the following condition: whenever $E \in \mathcal{D}$ and $E D_{0} \in J$, we have $E 1=0$.

Let $Q$ be an ideal of $\mathcal{D}$ that is maximal with respect to the following properties: $\mathcal{A} \subset Q$, and the system $S\left(D_{0}, Q\right)$ is solvable; that is, noncontradictory. There is such a maximal ideal, since being noncontradictory is a finite property; that is, if every finite subsystem of a system $\Sigma$ is noncontradictory, then so is $\Sigma$. It is enough to show that $S\left(D_{0}, Q\right)$ has a polynomial solution on $\mathbb{Z}^{n}$.

The function $D_{0} 1$ is constant. If its value is $c$ where $c \neq 0$, then let $q$ denote the constant $1 / c$ function. We show that $q$ is a solution of $S\left(D_{0}, Q\right)$. Clearly, $D_{0} q=1$. Let $f$ be a solution of $S\left(D_{0}, Q\right)$. If $D \in Q$, then $D 1=$ $D D_{0} f=D_{0}(D f)=0$, and thus $D q=0$. Therefore, the system $S\left(D_{0}, Q\right)$ has a polynomial solution, namely $q$, if $D_{0} 1 \neq 0$. So we may assume that $D_{0} 1=0$.

We prove that in this case every solution of the system $S\left(D_{0}, Q\right)$ is a polynomial. This will prove the statement, since, being noncontradictory, $S\left(D_{0}, Q\right)$ has a solution. 
We prove that $Q$ is a primary ideal. Since $\mathcal{D}$ is Noether, it is enough to show that $Q$ is irreducible; that is, $Q$ is not the intersection of two ideals strictly larger than $Q$. Suppose this is not true, and let $Q=Q_{1} \cap Q_{2}$, where $Q \subsetneq Q_{i}$ $(i=1,2)$. Since $Q$ is maximal, the systems $S\left(D_{0}, Q_{i}\right)$ are contradictory. Then there are difference operators $E_{i}$ such that $E_{i} D_{0} \in Q_{i}$ and $E_{i} 1 \neq 0(i=1,2)$. Clearly, $E_{1} 1, E_{2} 1$ are nonzero constant functions, and then $E_{1} E_{2} 1 \neq 0$.

Now $E_{i} D_{0} \in Q_{i}(i=1,2)$ implies $E_{1} E_{2} D_{0} \in Q_{1} \cap Q_{2}=Q$. Since $S\left(D_{0}, Q\right)$ is noncontradictory, this implies $E_{1} E_{2} 1=0$, a contradiction. Therefore, $Q$ is primary.

Let $J$ denote the ideal of $\mathcal{D}$ generated by the difference operators $\Delta_{h}(h \in$ $\left.\mathbb{Z}^{n}\right)$. Then we have $A 1=0$ for every $A \in J$. We saw above that $A 1=0$ holds for every $A \in Q$ as well, and thus $Q+J \neq \mathcal{D}$. Therefore, by a theorem of Krull [8, Theorem 2b, p. 145], we have $Q=\bigcap_{r=1}^{\infty}\left(Q+J^{r}\right)$. Since $D_{0} \notin Q$, it follows that $D_{0} \notin Q+J^{r}$ if $r$ is large enough. Fix such an $r$. We show that $J^{r} \subset Q$.

Suppose this is not true. Then, by the maximality of $Q, S\left(D_{0}, Q+J^{r}\right)$ is contradictory. Therefore, there is an $E \in \mathcal{D}$ such that $E D_{0} \in Q+J^{r}$ and $E 1 \neq 0$. Since $E 1$ is constant, we may assume that $E 1=1$.

Let $E=\sum_{i=1}^{k} a_{i} T_{b_{i}}$, where $a_{i} \in \mathbb{C}$ and $b_{i} \in \mathbb{Z}^{n}(i=1, \ldots, k)$. Then $E 1=1$ gives $\sum_{i=1}^{k} a_{i}=1$, and thus

$$
E-T_{0}=\sum_{i=1}^{k} a_{i}\left(T_{b_{i}}-T_{0}\right)=\sum_{i=1}^{k} a_{i} \Delta_{b_{i}} \in J
$$

and $\left(T_{0}-E\right)^{r} \in J^{r}$. Now $T_{0}-\left(T_{0}-E\right)^{r}=A E$ with a suitable $A \in \mathcal{D}$. Then we have

$$
\begin{aligned}
& \left(T_{0}-\left(T_{0}-E\right)^{r}\right) D_{0}=A E D_{0} \in Q+J^{r}, \\
& D_{0}=T_{0} D_{0}=A E D_{0}+\left(T_{0}-E\right)^{r} D_{0} \in Q+J^{r},
\end{aligned}
$$

which is impossible. This proves $J^{r} \subset Q$. In particular, we have $\Delta_{h_{1}} \ldots \Delta_{h_{r}} \in$ $Q$ for every $h_{1}, \ldots, h_{r} \in Q$.

Therefore, if $f$ is a solution of $S\left(D_{0}, Q\right)$, then $\Delta_{h_{1}} \ldots \Delta_{h_{r}} f=0$ for every $h_{1}, \ldots, h_{r} \in \mathbb{Z}^{n}$, and thus $f$ is a polynomial.

II. We prove that the statement of the theorem is true in the case when $G=\mathbb{Z}^{n}$.

Let $S$ be a noncontradictory system of equations $D_{i} f=p_{i}(i \in I)$, where $D_{i} \in \mathcal{D}$ and $p_{i} \in \mathbb{C}\left[x_{1}, \ldots, x_{n}\right]$ is a polynomial on $G$ for every $i \in I$. We prove that $S$ has a polynomial solution. It is clear that $S$ can be replaced by the set of all equations that can be obtained from $S$ by multiplying both sides of an equation of $S$ by an arbitrary difference operator, and adding the equations obtained. Therefore, we may assume that the set $J=\{D: \exists p,(D, p) \in S\}$ is an ideal. Since $\mathcal{D}$ is Noether, $J$ is generated by finitely many difference operators $D_{1}, \ldots, D_{m} \in J$. If $\left(D_{i}, p_{i}\right) \in J(i=1, \ldots, m)$, then the system $T=\left\{\left(D_{i}, p_{i}\right): i=1, \ldots, m\right\}$ is noncontradictory, and has the same solutions as $S$. It is enough to show that $T$ has a polynomial solution. 
Let $k$ be the smallest nonnegative integer such that the polynomials $p_{1}, \ldots, p_{m}$ only depend on the variables $x_{1}, \ldots, x_{k}$. If $k=0$ then by this condition we mean that $p_{1}, \ldots, p_{m}$ are constant functions.

We prove that $T$ has a polynomial solution by induction on $k$. First we assume that $k=0$; that is, $p_{1}, \ldots, p_{m}$ are constant functions.

The statement is clear if $p_{i}=0$ for every $i$. Otherwise, pick a $j$ such that $p_{j} \neq 0$. We may assume that $p_{j}=1$. Replacing the equation $D_{i} f=p_{i}$ by $\left(D_{i}-p_{i} \cdot D_{j}\right) f=0$ for every $i \neq j$ we obtain a system having the same solutions as $T$. Therefore, we may assume that $p_{i}=0$ for every $i \neq j$ and $p_{j}=1$. As we saw above, in this case the system has a polynomial solution, which proves the case $k=0$.

Let $0<k \leq n$, and suppose that the statement holds for $k-1$. Let $T=\left\{\left(D_{i}, p_{i}\right): i=1, \ldots, m\right\}$ be a noncontradictory system of equations on $\mathbb{Z}^{n}$ such that $p_{i} \in \mathbb{C}\left[x_{1}, \ldots, x_{k}\right]$ for every $i=1, \ldots, m$. We prove the following statement by induction on $d$ : if $p_{i} \in \mathbb{C}\left[x_{1}, \ldots, x_{k}\right]$ for every $i=1, \ldots, m$, $\sum_{i=1}^{m} \operatorname{deg} p_{i} \leq d$ and the system $D_{i} f=p_{i}(i=1, \ldots, m)$ is solvable, than it has a polynomial solution.

If $d=0$ then each $p_{i}$ is constant, and the statement has been proved above. Suppose that $d>0$, and that the statement is true for $d-1$. Let $e \in \mathbb{Z}^{n}$ denote the vector whose $k$ th coordinate is 1 and the other coordinates are zero. Then the system of equations $D_{i} f=\Delta_{e} p_{i}(i=1, \ldots, m)$ is solvable since $\Delta_{e} f$ is a solution whenever $f$ is a solution of $T$. We may assume that at least one of the polynomials $p_{i}$ is not constant. Then

$$
\sum_{i=1}^{m} \operatorname{deg} \Delta_{e} p_{i}<\sum_{i=1}^{m} \operatorname{deg} p_{i} \leq d .
$$

By the induction hypothesis, there is a polynomial $q$ such that $D_{i} q=\Delta_{e} p_{i}$ for every $i$. Let $Q \in \mathbb{C}\left[x_{1}, \ldots, x_{k}\right]$ be a polynomial such that $\Delta_{e} Q=q$, and consider the system $T^{\prime}$ of the equations $D_{i} f=r_{i}(i=1, \ldots, m)$, where $r_{i}=$ $p_{i}-D_{i} Q$ for every $i$. The system $T^{\prime}$ is solvable because $f_{0}-Q$ is a solution whenever $f_{0}$ is a solution of $T$.

Then $r_{i} \in \mathbb{C}\left[x_{1}, \ldots, x_{k}\right]$ and

$$
\Delta_{e} r_{i}=\Delta_{e} p_{i}-D_{i}\left(\Delta_{e} Q\right)=\Delta_{e} p_{i}-D_{i} q=0
$$

for every $i$. This implies that $r_{i}$ does not depend on the variable $x_{k}$ and, consequently, $r_{i} \in \mathbb{C}\left[x_{1}, \ldots, x_{k-1}\right]$ for every $i$. Therefore, by the induction hypothesis on $k$ we find that $T^{\prime}$ has a polynomial solution $P$. Then $P+Q$ is a polynomial solution of $T$. This proves the statement of the theorem in the case when $G=\mathbb{Z}^{n}$.

III. We prove that the statement of the theorem is true in the case when $G$ is finitely generated. In this case we have $G=F \times H$, where $F$ is finite, and $H$ is isomorphic to $\mathbb{Z}^{n}$ for a suitable $n$. We assume that $G$ is an inner product; 
that is, $F$ and $H$ are subgroups of $G$, and every element of $G$ can be written uniquely as $b+y$, where $b \in F$ and $y \in H$.

Let $S$ be a noncontradictory system of difference equations such that for every $(D, p) \in S$ the function $p$ is a polynomial on $G$. We prove that $S$ has a polynomial solution.

For every $f: G \rightarrow \mathbb{C}$ and $x \in G$ we denote by $\bar{f}(x)$ the average of the values $f(x+b)(b \in F)$. That is, let

$$
\bar{f}(x)=\frac{1}{|F|} \sum_{b \in F} f(x+b) \quad(x \in G) .
$$

Then $\bar{f}(x+b)=\bar{f}(x)$ for every $x \in G$ and $b \in F$. In other words, $\bar{f}$ is constant on every coset of $F$. We show that if $f$ is a solution of $S$, then so is $\bar{f}$.

If $p$ is a polynomial on $G$ then, for every $x \in G$ and $b \in F$ we have $p(x+b)=p(x)$. Indeed, the function $\phi(n)=p(x+n \cdot b)(n \in \mathbb{Z})$ is a polynomial on $\mathbb{Z}$. Since $b \in F$ is of finite order, $\phi$ is periodic, hence bounded. Thus $\phi$ is constant, and thus $\phi(1)=\phi(0)$ gives $p(x+b)=p(x)$. This gives $T_{b} p=p$ for every $b \in F$ and for every polynomial $p$.

Therefore, if $(D, p) \in S$ and $f$ is a solution of $S$, then $D f=p$, and

$$
\begin{aligned}
D \bar{f} & =D\left(\frac{1}{|F|} \sum_{b \in F} T_{b} f\right)=\frac{1}{|F|} \sum_{b \in F} D T_{b} f=\frac{1}{|F|} \sum_{b \in F} T_{b} D f \\
& =\frac{1}{|F|} \sum_{b \in F} T_{b} p=p .
\end{aligned}
$$

Thus $\bar{f}$ is a solution of $S$.

Let $\psi(b+y)=y$ for every $y \in H$ and $b \in F$. Then $\psi$ is a homomorphism from $G$ onto $H$. For every difference operator $D=\sum_{i=1}^{n} a_{i} T_{x_{i}}$ we denote by $\bar{D}$ the difference operator $\sum_{i=1}^{n} a_{i} T_{\psi\left(x_{i}\right)}$. The fact that $\bar{f}$ is constant on the cosets of $F$ implies that $\bar{D} \bar{f}=p$ for every $(D, p) \in S$. Therefore, the system $\bar{S}=\{(\bar{D}, p):(D, p) \in S\}$ has a solution, hence is not contradictory. Note that supp $\bar{D} \subset H$ for every difference operator $D$.

Since $H$ is isomorphic to $\mathbb{Z}^{n}$, it follows that $\bar{S}$ has a polynomial solution $\bar{q}$ on $H$. Let $q(x)=\bar{q}(\psi(x))$ for every $x \in G$. It is clear that $q$ is a polynomial on $G$. It is also clear that $q$ is a solution of $S$.

IV. Now we prove the theorem in the general case. Suppose $n=r_{0}(G)<\infty$. Let $h_{1}, \ldots, h_{n} \in G$ be independent elements of infinite order, and denote by $H$ the subgroup $\left\langle h_{1}, \ldots, h_{n}\right\rangle$. If $r_{0}(G)=0$, then we put $H=\{0\}$.

Let $S$ be a noncontradictory system of difference equations such that for every $(D, p) \in S$ the function $p$ is a polynomial on $G$. We prove that $S$ has a polynomial solution. Since $\operatorname{sc}\left(\mathcal{P}_{G}\right) \leq \omega$ by Theorem 6.1 , it is enough to show that every finite subsystem of $S$ has a polynomial solution. Therefore, we may assume that $S$ is finite. 
Let $F=\left\{h_{1}, \ldots, h_{n}\right\} \cup \bigcup_{(D, g) \in S} \operatorname{supp} D$. Then $F$ is finite, and $\langle F\rangle$ is finitely generated. By III, the system $S$ has a polynomial solution on $\langle F\rangle$. That is, there exists a polynomial $p \in \mathcal{P}_{\langle F\rangle}$ such that $D p(x)=g(x)$ for every $(D, g) \in S$ and $x \in\langle F\rangle$.

Since every additive function can be extended from $\langle F\rangle$ to $G$, it follows that $p$ can be extended to $G$ as a polynomial. Let $p_{1} \in \mathcal{P}_{G}$ be such an extension. If $(D, g) \in S$, then $D p_{1} \in \mathcal{P}_{G}, g \in \mathcal{P}_{G}$, and $D p_{1}=g$ on $\langle F\rangle$. Since $H \subset\langle F\rangle$, it follows from Lemma 2.3 that $D p_{1}=g$ on $G$. Since this is true for every $(D, g) \in S, p_{1}$ is a polynomial solution of $S$.

Remark 6.5. We note that the statement of Theorem 6.4 may be false in nondiscrete groups. For example, let $\alpha \in \mathbb{R}$ be irrational, and let $G$ denote the subgroup of $\mathbb{R}$ generated by 1 and $\alpha$ equipped with the subspace topology. Then $r_{0}(G)=2$.

Now the system of equations $S=\left\{\left(\Delta_{1}, 0\right),\left(\Delta_{\alpha}, 1\right)\right\}$ is noncontradictory, since solvable. On the other hand, it is easy to prove that $S$ does not have a continuous (even locally bounded) solution on $G$.

We note also that the statement of Theorem 6.4 can hold on a topological Abelian group $G$ only if $\operatorname{sc}\left(\mathcal{P}_{G}\right) \leq \omega$. Indeed, if $\operatorname{sc}\left(\mathcal{P}_{G}\right) \geq \omega_{1}$, then there is a system $S$ of difference equations on $G$ such that every finite subsystem has a polynomial solution, but $S$ does not have one. Then every finite subsystem of $S$ is solvable, hence so is $S$. If $(D, g) \in S$, then there is a polynomial $p$ such that $D p=g$, since $\{(D, g)\}$ is a finite subsystem of $S$. Thus $g=D p$ is a polynomial. Therefore, $S$ is a system satisfying the conditions of Theorem 6.4, but $S$ does not have a polynomial solution.

In particular, the statement of Theorem 6.4 is false if $G$ is a discrete Abelian group with $r_{0}(G)=\infty$. Indeed, in this case sc $\left(\mathcal{P}_{G}\right) \geq \omega_{1}$ by Theorem 6.1.

We prove, however, that if we require local polynomial solutions defined below, then Theorem 6.4 remains valid for countable Abelian groups.

We say that a continuous function $f: G \rightarrow \mathbb{C}$ is a local polynomial, if the restriction of $f$ to any finitely generated subgroup of $G$ is a polynomial.

Theorem 6.6. Let $S$ be a noncontradictory system of difference equations on the discrete and countable Abelian group $G$ such that for every $(D, g) \in S$ the function $g$ is a local polynomial on $G$. Then $S$ has a solution which is a local polynomial on $G$.

Proof. Let $\left\{x_{1}, x_{2}, \ldots\right\}$ be an enumeration of the elements of $G$. We denote by $G_{n}$ the subgroup $\left\langle x_{1}, \ldots, x_{n}\right\rangle$.

Let $S$ be a noncontradictory system of difference equations on $G$ such that for every $(D, g) \in S$ the function $g$ is a local polynomial on $G$. We prove that $G$ has a solution which is a local polynomial.

For every $n$ let $S_{n}$ denote the set of pairs $(D, g) \in S$ with $\operatorname{supp} D \subset G_{n}$. Then $S_{n}$ is noncontradictory, hence the system $S_{n}$ has a polynomial solution 
on the finitely generated group $G_{n}$. Let $A_{n}$ denote the set of polynomials $f$ defined on $G_{n}$ such that $f$ is a solution of $S_{n}$ on $G_{n}$. Then $A_{n}$ is an affine submodule of $\mathcal{P}_{G_{n}}$ by Lemma 2.1 .

If $n<k$, then $A_{n, k}=\left\{\left.f\right|_{G_{n}}: f \in A_{k}\right\}$ is also an affine submodule of $\mathcal{P}_{G_{n}}$. We show that $A_{n, k} \subset A_{n}$. Indeed, if $f \in A_{k}$, then $f$ is a polynomial solution of $S_{k}$ on $G_{k}$. Since $G_{n} \subset G_{k}$ and $S_{n} \subset S_{k}$, it is clear that $\left.f\right|_{G_{n}}$ is a solution of $S_{n}$; that is, $\left.f\right|_{G_{n}} \in A_{n}$.

Therefore, $A_{n}, A_{n, n+1}, A_{n, n+2}, \ldots$ is a nested sequence of affine submodules of $\mathcal{P}_{G_{n}}$. By Theorem 2.2, there is an $N$ such that $A_{n, N}=A_{n, k}$ for every $k>N$. We put $\bar{A}_{n}=A_{n, N}$. Then $\bar{A}_{n}$ is an affine submodule of $\mathcal{P}_{G_{n}}$ satisfying

$$
\bar{A}_{n} \subset\left\{\left.f\right|_{G_{n}}: f \in \bar{A}_{k}\right\}
$$

for every $n<k$. Let $f_{1} \in \bar{A}_{1}$ be arbitrary. By (5), there is an $f_{2} \in \bar{A}_{2}$ such that $\left.f_{2}\right|_{G_{1}}=f_{1}$. Then, applying (5) again, we obtain an $f_{3} \in \bar{A}_{3}$ such that $\left.f_{3}\right|_{G_{2}}=f_{2}$. Continuing the process we find $f_{n} \in \bar{A}_{n}$ for every $n$ such that $\left.f_{k}\right|_{G_{n}}=f_{n}$ for every $n<k$.

Let $f$ be the unique common extension of the functions $f_{n}$ to $G$. We prove that $f$ is a solution of $S$. Let $(D, p) \in S$ and $x \in G$ be arbitrary. Then there is an $n$ such that $\operatorname{supp} D \subset G_{n}$ and $x \in G_{n}$. Then, as $(D, p) \in S_{n}$ and $f_{n}$ is a solution of $S_{n}$ on $G_{n}$, we have $D f_{n}(x)=p(x)$. Therefore, $D f(x)=p(x)$ for every $(D, p) \in S$ and $x \in G$; that is, $f$ is a solution of $S$.

If $H$ is a finitely generated subgroup of $G$, then there is an $n$ such that $H \subset G_{n}$. Since $f_{n}$ is a polynomial on $G_{n}$, it follows that $f$ is a polynomial on $G_{n}$, hence on $H$. This proves that $f$ is a local polynomial.

We do not know whether or not the statement of Theorem 6.6 is true for every discrete Abelian group $G$.

\section{Projections onto $\mathcal{P}_{G}$}

Let $\Phi: \mathbb{C}^{\mathbb{R}} \rightarrow \mathbb{C}[x]$ be a linear map. It was proved in $[4$, Theorem 5.2$]$ that if there is a polynomial $p$ such that $\Phi(p) \neq 0$, then the group

$$
G_{\Phi}=\left\{a \in \mathbb{R}: \Phi\left(T_{a} f\right)=T_{a} \Phi(f) \text { for every } f \in \mathbb{C}^{\mathbb{R}}\right\}
$$

is countable, moreover, $G_{\Phi}=\{c \cdot r: r \in \mathbb{Q}\}$ for some $c \in \mathbb{R}$. In particular, if $\Phi$ is a projection from $\mathbb{C}^{\mathbb{R}}$ onto $\mathbb{C}[x]$; that is, if $\Phi(p)=p$ for every $p \in \mathbb{C}[x]$, then $G_{\Phi}$ must be of this form.

On the other hand, it was shown in [4, Theorem 5.3] that there exists a projection $\Phi$ from $\mathbb{C}^{\mathbb{R}}$ onto $\mathbb{C}[x]$ such that $G_{\Phi}=\mathbb{Q}$. As the next theorem shows, the existence of a projection of all functions defined on a topological Abelian group $G$ onto $\mathcal{P}_{G}$ commuting with a given group of translations depends on the solvability of certain systems of difference equations in polynomials. 
Theorem 7.1. Let $G$ be a topological Abelian group, and let $H$ be a subgroup of $G$. Then the following are equivalent.

(i) There exists a projection $\Phi$ from $\mathbb{C}^{G}$ onto $\mathcal{P}_{G}$ such that $\Phi\left(T_{a} f\right)=T_{a} \Phi(f)$ for every $f \in \mathbb{C}^{G}$ and $a \in H$.

(ii) If $S$ is a noncontradictory system of difference equations on $G$ such that $\operatorname{supp} D \subset H$ and $g \in \mathcal{P}_{G}$ for every $(D, g) \in S$, then $S$ has a polynomial solution.

Proof. Suppose (i), and let $S$ be a system as in (ii). Since $S$ is noncontradictory, it has a solution $f \in \mathbb{C}^{G}$. Let $q=\Phi(f)$, then $q \in \mathcal{P}_{G}$. We show that $q$ is a solution of $S$. Indeed, if $(D, g) \in S$, then $\operatorname{supp} D \subset H$. Since $\Phi$ commutes with translations by elements of $H$ and $D$ is a linear combination of such translations, it follows that $\Phi$ commutes with $D$. Then we have

$$
D q=D \Phi(f)=\Phi(D f)=\Phi p=p,
$$

and thus $q$ is indeed a solution of $S$.

Next suppose (ii). We construct a projection $\Phi$ satisfying the conditions of (i).

Let $\mathcal{W}$ denote the set of pairs $(V, \Phi)$ such that $V$ is a linear subspace of $\mathbb{C}^{G}$, $\mathcal{P}_{G} \subset V, V$ is invariant under translations by elements of $H$, and $\Phi: V \rightarrow \mathcal{P}_{G}$ is a projection commuting with translations by elements of $H$. Then $\mathcal{W}$ is nonempty, as $\left(\mathcal{P}_{G}\right.$, identity $) \in \mathcal{W}$. We define a partial order on $\mathcal{W}$ by writing $\left(V_{1}, \Phi_{1}\right) \leq\left(V_{2}, \Phi_{2}\right)$ if $V_{1} \subset V_{2}$ and $\Phi_{2}$ is an extension of $\Phi_{1}$. By Zorn's lemma there is a maximal $(V, \Phi) \in \mathcal{W}$. In order to prove the theorem, it is enough to show that $V=\mathbb{C}^{G}$. Suppose this is not true, and let $f_{0} \in \mathbb{C}^{G} \backslash V$. Then

$$
V^{*}=\left\{v+D f_{0}: v \in V, D \in \mathcal{D}_{G}, \operatorname{supp} D \subset H\right\}
$$

is a linear subspace of $\mathbb{C}^{G}$ that contains $V \cup\left\{f_{0}\right\}$ and is invariant under translations by elements of $H$.

We shall prove that $\Phi$ can be extended to $V^{*}$ as a linear operator commuting with translations by elements of $H$. Since $(V, \Phi)$ is maximal, this will be a contradiction, proving the theorem. Let

$$
S=\left\{(D, p): D \in \mathcal{D}_{G}, \operatorname{supp} D \subset H, D f_{0} \in V, p=\Phi\left(D f_{0}\right)\right\} .
$$

We show that the system $S$ is non-contradictory. By [4, Lemma 2.3], it is enough to show that if $A_{i} \in \mathcal{D}_{G}$, supp $D \subset H$ and $\left(D_{i}, p_{i}\right) \in S(i=1, \ldots, n)$, then $\sum_{i=1}^{n} A_{i} D_{i}=0$ implies $\sum_{i=1}^{n} A_{i} p_{i}=0$. Since $\Phi$ commutes with difference operators whose support is a subset of $H$, we have

$$
\begin{aligned}
\sum_{i=1}^{n} A_{i} p_{i} & =\sum_{i=1}^{n} A_{i} \Phi\left(D_{i} f_{0}\right)=\sum_{i=1}^{n} \Phi\left(A_{i} D_{i} f_{0}\right)=\Phi\left(\left(\sum_{i=1}^{n} A_{i} D_{i}\right) f_{0}\right) \\
& =\Phi(0)=0 .
\end{aligned}
$$

Therefore, $S$ is non-contradictory, as we stated. By condition (ii), $S$ has a polynomial solution. Let $q$ be such a solution; then $D q=p$ for every $(D, p) \in S$. 
Let $\Phi^{*}\left(v+D f_{0}\right)=\Phi(v)+D q$ for every $v \in V$ and $D \in \mathcal{D}_{G}$ with supp $D \subset H$. It is easy to check that $\Phi^{*}$ is a well-defined extension of $\Phi$ and commutes with translations by elements of $H$.

Corollary 7.2. Let $G$ be a topological Abelian group. There exists a projection from $\mathbb{C}^{G}$ onto $\mathcal{P}_{G}$ commuting with all translations if and only if $\operatorname{sc}\left(\mathcal{P}_{G}\right) \leq \omega$, and whenever $S$ is a finite and noncontradictory system of difference equations on $G$ such that $g \in \mathcal{P}_{G}$ for every $(D, g) \in S$, then $S$ has a polynomial solution.

Proof. We have to show that if $H=G$, then (ii) of Theorem 7.1 is equivalent to the condition formulated in the theorem.

Suppose that (ii) of Theorem 7.1 holds. If $S$ is a system of difference equations such that every finite subsystem of $S$ has a polynomial solution, then $S$ is necessarily noncontradictory, and thus $S$ has a polynomial solution by (ii). Therefore, we have $\operatorname{sc}\left(\mathcal{P}_{G}\right) \leq \omega$. From this observation the statement of the corollary easily follows.

Comparing this with Theorem 6.4 we obtain the following.

Corollary 7.3. If $G$ is a discrete Abelian group with $r_{0}(G)<\infty$, then there exists a projection from $\mathbb{C}^{G}$ onto $\mathcal{P}_{G}$ commuting with all translations.

Returning to projections commuting with some translations, we prove that $\left[4\right.$, Theorem 5.3] can be generalized to $\mathbb{R}^{n}$.

Theorem 7.4. There exists a projection from $\mathbb{C}^{\mathbb{R}^{n}}$ onto $\mathbb{C}\left[x_{1}, \ldots, x_{n}\right]$ commuting with translations by vectors having rational coordinates.

Proof. It is enough to show that condition (ii) of Theorem 7.1 is satisfied if $G=$ $\mathbb{R}^{n}$ and $H=\mathbb{Q}^{n}$. Let $S$ be a noncontradictory system of difference equations on $\mathbb{R}^{n}$ such that $\operatorname{supp} D \subset \mathbb{Q}^{n}$ and $g \in \mathbb{C}\left[x_{1}, \ldots, x_{n}\right]$ for every $(D, g) \in S$. We have to show that $S$ has a polynomial solution. Since $\operatorname{sc}\left(\mathbb{C}\left[x_{1}, \ldots, x_{n}\right]\right)=\omega$ by Theorem 5.1, it is enough to prove that every finite subsystem of $S$ has a polynomial solution.

Therefore, we may assume that $S$ is finite. Then there is a positive integer $N$ such that for every $(D, g) \in S, \operatorname{supp} D$ consists of vectors of the form $\left(a_{1} / N, \ldots, a_{n} / N\right)$, where $a_{1}, \ldots, a_{n}$ are integers. For every $f: \mathbb{R}^{n} \rightarrow \mathbb{C}$ we denote by $f^{\prime}$ the function $x \mapsto f(x / N)\left(x \in \mathbb{R}^{n}\right)$. Note that $f$ is a polynomial if and only if $f^{\prime}$ is.

If $(D, g) \in S$, where $D=\sum_{i=1}^{k} c_{i} T_{b}$, then we put $D^{\prime}=\sum_{i=1}^{k} c_{i} T_{N \cdot b}$. It is clear that a function $f: \mathbb{R}^{n} \rightarrow \mathbb{C}$ is a solution of $S$ if and only if $f^{\prime}$ is a solution of the system $S^{\prime}=\left\{\left(D^{\prime}, g^{\prime}\right):(D, g) \in S\right\}$. Since $S$ is solvable by assumption, it follows that $S^{\prime}$ is also solvable. Note that supp $D^{\prime} \subset \mathbb{Z}^{n}$ and $g^{\prime} \in \mathbb{C}\left[x_{1}, \ldots, x_{n}\right]$ for every $\left(D^{\prime}, g^{\prime}\right) \in S^{\prime}$. If we show that $S^{\prime}$ has a polynomial solution, the proof will be complete.

By Theorem $6.4, S^{\prime}$ has a polynomial solution on $\mathbb{Z}^{n}$. Since the polynomials on $\mathbb{Z}^{n}$ are the elements of $\mathbb{C}\left[x_{1}, \ldots, x_{n}\right]$ restricted to $\mathbb{Z}^{n}$, we find that there 
exists a polynomial $p \in \mathbb{C}\left[x_{1}, \ldots, x_{n}\right]$ such that $D^{\prime} p(x)=g^{\prime}(x)$ for every $x \in \mathbb{Z}^{n}$ and $\left(D^{\prime}, g^{\prime}\right) \in S^{\prime}$. This implies that if $\left(D^{\prime}, g^{\prime}\right) \in S^{\prime}$, then $D^{\prime} p(x)=g^{\prime}(x)$ holds for every $x \in \mathbb{R}^{n}$ as well. Indeed, $D^{\prime} p-g^{\prime}$ is a polynomial of $n$ variables that vanishes on $\mathbb{Z}^{n}$. Now, if a polynomial $q \in \mathbb{C}\left[x_{1}, \ldots, x_{n}\right]$ vanishes on $\mathbb{Z}^{n}$, then it vanishes on $\mathbb{R}^{n}$ by Lemma 2.3. Thus $D^{\prime} p-g^{\prime}=0$ on $\mathbb{R}^{n}$ for every $\left(D^{\prime}, g^{\prime}\right) \in S^{\prime}$. Therefore, $p$ is a polynomial solution of $S^{\prime}$.

\section{Funding Open access funding provided by Eötvös Loránd University.}

Open Access. This article is licensed under a Creative Commons Attribution 4.0 International License, which permits use, sharing, adaptation, distribution and reproduction in any medium or format, as long as you give appropriate credit to the original author(s) and the source, provide a link to the Creative Commons licence, and indicate if changes were made. The images or other third party material in this article are included in the article's Creative Commons licence, unless indicated otherwise in a credit line to the material. If material is not included in the article's Creative Commons licence and your intended use is not permitted by statutory regulation or exceeds the permitted use, you will need to obtain permission directly from the copyright holder. To view a copy of this licence, visit http:// creativecommons.org/licenses/by/4.0/.

Publisher's Note Springer Nature remains neutral with regard to jurisdictional claims in published maps and institutional affiliations.

\section{References}

[1] Elekes, M., Laczkovich, M.: A cardinal number connected to the solvability of systems of difference equations in a given function class. J. Anal. Math. 101, 199-218 (2007)

[2] Elekes, M., Kiss, V., Vidnyánszky, Z.: Ranks on the Baire class $\xi$ functions. Trans. Am. Math. Soc. 368(11), 8111-8143 (2016)

[3] Hewitt, E., Ross, K.A.: Abstract Harmonic Analysis, vol. I, 2nd edn. Springer, Berlin (1979)

[4] Laczkovich, M.: Operators commuting with translations, and systems of difference equations. Colloq. Math. 80, 1-22 (1999)

[5] Laczkovich, M.: Polynomial mappings on Abelian groups. Aequationes Math. 68, 177-199 (2004)

[6] Laczkovich, M.: Vector valued polynomials, exponential polynomials and vector valued harmonic analysis. Res. Math. 75(4), article 150 (2020)

[7] Laczkovich, M., Székelyhidi, L.: Spectral synthesis on discrete Abelian groups. Math. Proc. Camb. Philos. Soc. 143, 103-120 (2007)

[8] van der Waerden, B.L.: Algebra, vol. II. Springer, Berlin (1991)

\section{Miklós Laczkovich}

ELTE Eötvös Loránd University

Budapest

Hungary

e-mail: miklos.laczkovich@gmail.com

Received: October 21, 2020

Revised: May 25, 2021

Accepted: May 26, 2021 